\title{
Il fenomeno della sofferenza di padre Pio da Pietrelcina descritto nelle lettere al direttore spirituale padre Benedetto
}

Nel presente articolo voglio presentare i fenomeni della sofferenza, descritti da Padre Pio da Pietrelcina nelle lettere al suo direttore spirituale padre Benedetto da San Marco ${ }^{1}$ in Lamis. Usando il metodo teologico, cercerò di cogliere dall'Epistolario di padre Pio i tratti distintivi del dolore fisico e della sofferenza spirituale o morale per analizzarli. Questa tematica è nuova, perciò non ci sono ancora i studi dedicati escluivamente ad essa. Piano del lavoro è sequente. Nel primo paragrafo presenterò, come una sorta di base "letteraria", la breve caratteristica dell'Epistolario I, ponendo l'attenzione ai tre elementi: l'autore e il destinatario dell'epistolario e la corrispondenza tra di loro. Nel secondo paragrafo mostrerò il fenomeno della sofferenza, come la nozione generale, da due prospettive: i dolori fisici e la sofferenza spirituale o morale. Vorrei descrivere le sfumature dei dolori fisici, raggruppandoli in classi: la prima, della causa naturale, cioè le malattie e la seconda,

1 Padre Benedetto Nardella (1872-1942), cappuccino italiano della Provincia di Foggia. E' stato scelto come direttore spirituale da stesso padre Pio nel 1905 e lo è rimasto fino al 1922. Altre notizie su padre Benedetto si trovano nel presente articolo nel § 1.2. Padre Benedetto, il destinatario delle lettere. 
della causa "sopranaturale", dell'origine diabolica, come le vessazioni diaboliche e dell'origine divina come la transverberazione del costato e del cuore ed anche le stimmate. Alla fine caratterizzerò la sofferenza spirituale o morale "in genere", e pur particolare, conseguendo con la descrizione della "notte oscura" ed indicando alcune cause delle sofferenze spirituali, come timori e tentazioni.

\section{Epistolario I: l'autore, il destinatario e la corrispondenza}

L'Epistolario I. Corrispondenza con i direttori spirituali (1910-1922) è un volume chiave per la conoscenza di padre Pio, un volume-base per scriverne la vita e mettere in debita evidenza le eccezionali ricchezze della sua anima ${ }^{2}$. La corrispondenza epistolare è la buona testimonianza della sua propria e distintiva personalità, proiettando "una luce più divina che umana, sulla figura spirituale del protagonista, perché contiene la rivelazione di esperienze mistiche sopranaturali" ${ }^{3} \mathrm{e}$ manifesta le sue caratteristiche psico-fisiologiche. L'epistolario ha evidenziato in padre Pio una notevole capacità di scrittore. L'umile frate da Pietrelcina si farà apprezzare come lo scrittore con "lo spirito del letterato, l'animo dell'artista, il cuore del poeta" facendo apparire la ricchezza dei suoi pensieri e la profondità delle esperienze mistiche. Sul cammino spirituale il mistico sofferente sarà accompagnato da padre Benedetto, che rimane il suo direttore spirituale, il maestro più autorevole e decisivo, a cui sono indirizzati personalmente i resoconti di coscienza più importanti e più minuziosi. La corrispondenza epistolare ci mostrerà il modo e la frequenza dei rapporti intercorsi tra padre Pio e padre Benedetto e ce ne chiarirà anche i motivi.

2 Padre Pio da Pietrelcina, Epistolario I. Corrispondenza con i direttori spirituali (1910-1922), a cura di M. da Pobladura e A. da Ripabottoni, San Giovanni Rotondo $1995^{3}$.

3 L. Ciappi, Padre Pio alla luce del suo epistolario, in Congregatio de Causis Sanctorum, Sipontina, Beatificationis et Canonizationis Servi Dei Pii a Pietrelcina, sacerdotis professi OFM Cap. (Francesco Forgione. Pietrelcina 1887 - San Giovanni Rotondo 1968), Positio super virtutibus, Biografia documentata, III/2, Romae 1997, p. 246.

A. D’Errico, Padre Pio scrittore, a cura di G. Di Flumeri, San Giovanni Rotondo 1984, p. 85. 


\subsection{Padre Pio autore dell'Epistolario I}

Voglio esordire con un passo tratto dalla presentazione di padre Pio come l'autore dell'Epistolario I. Nel primo paragrafo descrivo la personalità del religioso cappuccino mediante le caratteristiche psico-fisiologiche, quelle che fanno emergere la sua ricchezza affettiva, dimostrando l'esteriorizzazione dei sentimenti amorevoli e le manifestazioni scostanti, che testimoniano il suo sistema nervoso molto sensibile e notevolmente delicato, elencando alcune caratteristiche della sua sfera fisiologica e psichica. Nel secondo paragrafo, invece, faccio un abbozzo del suo carattere come scrittore. Vorrei esaminare le lettere di padre Pio, sotto l'aspetto di scrittore, indicando i seguenti elementi: la conoscenza delle varie lingue, le norme generali nello scrivere le lettere, le difficoltà ad esprimere l'esperienza mistica, gli eidola lessicali, il tono, lo stile e la sensibilità poetica.

Prima voglio considerare, in modo generale, tutto ciò che costituisce il carattere personale di padre Pio come mistico e come autore dell'Epistolario I. Supponiamo che non sia possibile conoscere il suo carattere mistico, se non lo si studia nella sua concretezza, tenendo conto delle sue doti e dei suoi difetti naturali:

Il mistico, nella sua concretezza e nei suoi stati particolari, non si spoglia, né del suo temperamento, né delle sue infermità fisiche, né dei suoi nervi, né della sua cultura intellettuale o morale, né della sua recettività rispetto agli influssi ambientali, in una parola, né della sua fisiologia, né della sua psicologia ${ }^{5}$.

La corrispondenza è ricca di rilievi e sfumature, che riecheggiano, da una parte, le caratteristiche umane di padre Pio, svelando parzialmente il suo carattere e temperamento ${ }^{6}$ e, dall'altra, il lento lavorio della grazia che perfeziona la natura. Vi sono varie classificazioni dei tempe-

5 J. Maréchal, Études sur la Psichologie des Mystiques, vol. I, Paris 1938 (trad. it. a cura di E. Balducci), p. 173.

6 Cf. Marcozzi V., Considerazioni sui fatti straordinari di Padre Pio, in Congregatio De Causis Sanctorum, Sipontina, Beatificationis et Canonizationis Servi Dei Pii a Pietrelcina, sacerdotis professi OFM Cap. (Francesco Forgione. Pietrelcina 1887 - San Giovanni Rotondo 1968), Quaestiones selectae, vol. IV, Bari, 1997, p. 221. 
ramenti. Cercho di studiare le caratteristiche psico-fisiologiche del religioso cappuccino, manifestate nell'Epistolario I. Corrispondenza con $i \mathrm{di}$ rettori spirituali (1910-1922).

La ricchezza affettiva di padre Pio è superiore alla norma: lo dimostra, sia nell'esteriorizzazione dei sentimenti amorevoli, sia, nonostante il freno che impiega, alle volte, nelle manifestazioni aggressive, scostanti ${ }^{7}$. Le manifestazioni amorevoli si riscontrano nelle espressioni affettive ai direttori spirituali, per esempio: "non vi mando un bacio poiché è troppo poco per le fatiche sostenute per me, ma sibbene vi mando tutto quello che ho nel cuore per voi ossia una tenerezza infinita" e nel modo in cui manda gli auguri per l'onomastico e le feste pasquali e natalizie ai direttori, manifestando la sua cordiale amicizia e gratitudine ${ }^{9}$. Conosciamo gli atteggiamenti scostanti attraverso la loro descrizione fatta da padre Pio e quando egli si rammarica, nonostante i persistenti sforzi, di non riuscire a controllarsi: "Mi rammarico che, senza volerlo e senza avvertirlo, qualche volta mi accade di alzare un po' la voce in ciò che riguarda la correzione" ${ }^{10}$ oppure confessa con sincerità: "senza che lo voglia, vado soggetto ad atti d'impazienza"11. Il religioso si presenta anche come uomo impetuoso, ma questi scatti d'ira non erano colpevoli, perché causati spesso, se non sempre, da motivi sopranaturali. Scrive al padre Benedetto:

Il tutto si compendia in questo: sono divorato dall'amore di Dio e dall'amore del prossimo. [...] Credetemi pure, padre, delle sfuriate, che alle volte ho fatto, sono causate proprio da questa dura prigionia, chiamiamola pure fortunata ${ }^{12}$.

A una forte emotività, padre Pio accompagnava un sistema nervoso molto sensibile e notevolmente delicato, risentendo di alcuni effetti poco desiderabili, sia nella sfera fisiologica che in quella psichica ${ }^{13}$. Nella sfe-

Cf. V. Marcozzi, Considerazioni sui fatti straordinari di Padre Pio, in QS, 221.

Epist. I, 256.

Cf. Epist. I, 344; 491; 1250.

Epist. I, 1170.

Epist. I, 883.

Epist. I, 1247.

Cf. V. Marcozzi, Considerazioni suifatti straordinari di Padre Pio, in QS, 224. 
ra fisiologica si notano: prostrazioni e debolezza ${ }^{14}$, mal di capo con frequenza ${ }^{15}$, intensi sudori ${ }^{16}$ e vomiti ${ }^{17}$. Quest'argomento lo vogliamo sviluppare nella seconda parte di questo capitolo, nel paragrafo dedicato alle malattie di padre Pio. Nella sfera psichica è frequente l'ansia o l'angoscia ${ }^{18}$ e lo scrupolo ${ }^{19}$. Vediamo queste affermazioni nei successivi paragrafi che trattano delle differenti cause della sofferenza spirituale. Le caratteristiche psico-fisiologiche di padre Pio con la sua ricchezza affettiva e con il suo sistema nervoso molto sensibile e notevolmente delicato si evidenziano dalla corrispondenza ai direttori spirituali. Ci siamo fermati brevemente su ciascuna di esse, ponendo l'attenzione all'esteriorizzazione dei sentimenti amorevoli e alle manifestazioni scostanti, ed elencando alcuni effetti nella sfera fisiologica e nella psichica.

In sequito rivolgeremo l'attenzione sugli elementi della sua produzione letteraria come: la conoscenza delle varie lingue, le norme generali nello scrivere le lettere, le difficoltà ad esprimere l'esperienza mistica, gli eidola lessicali, il tono, lo stile e la sensibilità poetica. Dobbiamo anche ricordare che la sua attività letteraria abbraccia soltanto dodici anni, dal 1910 al 1922. Questi sono i primi della sua vita sacerdotale e rappresentano una piccola parte della sua vita naturale e spirituale durata ottantuno anni. All'inizio, voglio accennare alla conoscenza delle varie lingue da parte dell'autore dell'Epistolario I. Nell'iter scolastico padre Pio non aveva studiato né francese, né greco, noi non sappiamo come e quando egli da autodidatta studiò queste lingue, ma, proprio negli anni 1912-1915, padre Agostino gli scriveva le lettere ora in francese, ora in greco ${ }^{20}$. Il religioso riferisce che il suo angelo custode gli traduceva il greco e gli faceva "anche da maestro nella spiegazione di altre lingue" 21 e in qualche lettera risponde addirittura in francese corret-

\footnotetext{
Cf. Epist. I, 230; 483; 490; 495.

Cf. Epist. I, 381; 583; 797.

Cf. Epist. I, 180.

Cf. Epist. I, 190.

Cf. Epist. I, 756-759.

Cf. Epist. I, 185-186; 278.

Cf. Epist. I, in nota (2), 302; 304.

Cf. Epist. I, 304.
} 
to ${ }^{22}$. Padre Pio conosceva bene il latino e lo deduciamo non soltanto dalla quantità e qualità delle citazioni e dei riferimenti, ma anche dalla lettura delle sue lettere, dove "questa lingua fu il punto di riferimento mentale, quanto a termini, moduli e impianti strutturali della sua prosa: il suo italiano risente e sa di latino"23.

Nelle lettere di padre Pio troviamo le espressioni tolte dal dialetto parlato e italianizzate. Egli amava esprimersi talora nel suo dialetto e l'italiano che scriveva era agile e semplice. La norma che segue generalmente nello scrivere le sue lettere è "realizzare chiaro e limpido con la tensione necessitante del sentimento, senza retorica, senza intenzionalità celebrarli d'effetti" ${ }^{24}$. Così, la lettera del 7 aprile 1915 a padre Benedetto è dominata da fervido rigore logico e conoscenza della terminologia della logica, in una forma che appare severamente controllata: dall'uso degli aggettivi (“...se non con animo ilare, almeno con animo forte"; "cordialissimi ringraziamenti ed infinite benedizioni, con solenne promessa") a certi costrutti cui sottostanno strutture latine ("questa di lui assistenza"; "veggo inferiore essere il mio amore") ${ }^{25}$. I mistici di tutti tempi hanno lottato sempre con l'insufficienza del vocabolario per esprimere le loro mistiche elevazioni e la straordinaria esperienza dell'unione con Dio. Le idee e i pensieri oltrepassano i limiti della parola e della penna. Padre Pio aveva lo stesso problema. Quando, nelle lettere ai suoi direttori spirituali, deve esprimere la sua esperienza mistica, egli avverte e lamenta l'insufficienza del vocabolario e, comunque, dell'umano linguaggio: “Mi vaggo quasi nell'assoluta impossibilità di poter esprimere l'operazione del diletto" 26 .

In ogni scrittore ricorrono termini con alto indice di frequenza. Questi termini, gli eidola lessicali, costituiscono la chiave più sicura per scoprire i più riposti sentimenti che vibrano nell'animo di colui che li adopera. Nell'Epistolario I di padre Pio tra i termini che presentano il più alto indice di frequenza troviamo: Gesù, Dio, il celeste Padre, Croce, de-

Cf. Epist. I, 275; 284; 300; 301; 315.

Cf. A. D’Errico, Padre Pio scrittore, 20.

Cf. A. D'Errico, Padre Pio scrittore, 25.

Cf. A. D’Errico, Padre Pio scrittore, 30-31; Cf. Epist. I, 555-558.

Epist. I, 1112; Cf. Epist. I, 265. 
solazione, agonia, trafiggere, martirio, sofferenza, pene, dolore, amore, carità, notte (alta, tetra, oscura: dell'anima), buio, tenebre, lamenti, lacrime, fiat ${ }^{27}$. Questi termini punteggianti l'Epistolario I gli venivano indubbiamente con immediatezza e da soli bastano a fare luce profonda sulle tormentose e amare sofferenze della sua anima oltre del suo fisico. In molte lettere che hanno tutta l'aria di essere delle esposizioni o meglio delle confessioni, dal tono semplice, improvvisi, scattano sovente il colpo d'ala e l'impennata lirica. Ad esempio, nella lettera del 6 gennaio 1917 a padre Benedetto, il cui tessuto generale è di tono assai umile, improvvisamente la prosa s'eleva alta e ardita, in una descrizione realistica e drammatica:

Fitte tenebre coinvolgono il mio spirito, ed altro non veggo se non buio e sempre buio, altro non vi scorgo se non il movimento di fiere, che minacciano di farmi loro preda; altro non ascolta il mio udito se non il continuo ruggito di dette fiere, che mi fanno morire i tutti i momenti per lo spavento ${ }^{28}$.

Le lettere sono scritte in stile piano e semplice, alle volte anche disadorno e non privo di errori grammaticali e lessicali. Anche se a volte stende la minuta è evidente che non lima letterariamente quanto ha scritto. Però i suoi scritti non sono privi d'immagine ed espressioni eleganti e poetiche. Padre Pio nelle sue lettere adopera "immagini semplici, estratte dalla concreta esperienza e dalla viva realtà della vita e della natura, coloro che compaiono spesso le suggestioni poetiche"29. L'Epistolario I presenta il tessuto dell'esposizioni punteggiato da "suggestive immagini liriche, autentici aliti di poesia, ora delicati ora vibranti"30. Padre Pio, nella lettera del 20 giugno 1915 a padre Agostino, presenta una definizione della fede cristiana:

[...] fiore che Iddio pietoso fa sbocciare tra le spine del dolore, innaffiato dalle lagrime dell'anima che pazientemente soffre, che umilmente ai divini voleri si uniforma e che caldamente e fervidamente prega ${ }^{31}$.

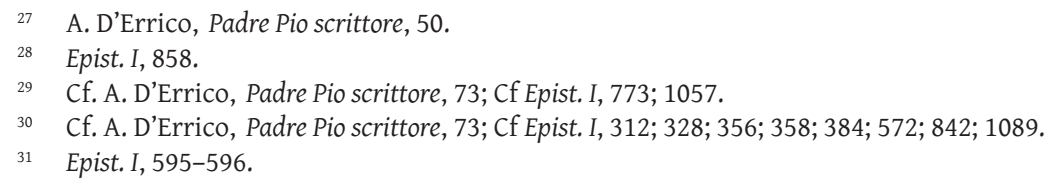


Le lettere del religioso, come quella già presentata prima, mostrano sempre un tono di vivo e profondo calore umano sulle sofferenze altrui e sono dettate da vivo slancio d'amore. Padre Pio, sotto l'aspetto di scrittore, viene a collocarsi tra i grandi scrittori di ascetica e di mistica non soltanto per il contenuto dei suoi scritti, ma anche per quelle possibilità espressive ${ }^{32}$. Possiamo aggiungere che nell'Epistolario I vi è un gruppo di lettere di una straordinaria bellezza letteraria e spirituale, degne di comparire in una antologia di spiritualità. Questo gruppo degli scritti contiene i più complessi stati della vita mistica descritti con meravigliosa limpidezza di linguaggio e con comune chiarezza di concetti.

Condivido pienamente i giudizi del prof. Alfonso D'Errico che presenta il religioso cappuccino sotto l'aspetto di scrittore, riportando il seguente brano dal suo libro Padre Pio scrittore, che raccoglie in sintesi il pensiero dell'autore:

Padre Pio non esercitò il mestiere del letterato e dell'artista, pur non mancandogliene, come ho dimostrato, possibilità e mezzi, in particolare acutezza di osservazione, intensità di sentimento, sensibilità poetica e il necessario possesso del mezzo espressivo. Ed è una magnifica realtà che a padre Pio, che tutte le sue ore impegnava in dolorosa e sofferta opera di corredenzione, piaceva anche l'espressione precisa, fiorita, letterariamente solida, dignitosamente artistica: e questi dotti, da lui possedute, confluivano tanto più spontanee e immediate nella pagina, quanto più intenso era il suo sentimento, più acuti i moti che s'agitavano nell'anima. Il che vuol dire che padre Pio aveva lo spirito del letterato, l'animo dell'artista, il cuore del poeta ${ }^{33}$.

Padre Pio scrittore con "lo spirito del letterato, l'animo dell'artista e il cuore del poeta" ci mostra la ricchezza dei suoi pensieri e la profondità delle esperienze mistiche. In questo paragrafo, dedicato a padre Pio autore dell'Epistolario I, abbiamo seguito la personalità del religioso attraverso le caratteristiche psico-fisiologiche, accennando alla sua affettività e al suo sistema nervoso e, poi, abbiamo fatto un abbozzo del suo carattere come scrittore. Padre Pio, con la sua produzione letteraria, è stato esaminato sotto i seguenti aspetti: la conoscenza delle varie

${ }^{32}$ Questo è il giudizio che presenta padre Gerardo di Flumeri e prof. Alfonso d'Errico; Cf. A. D’Errico, Padre Pio scrittore, 17-18.

33 A. D’Errico, Padre Pio scrittore, 85. 
lingue, le norme generali nello scrivere le lettere, le difficoltà ad esprimere l'esperienza mistica, gli eidola lessicali, il tono, lo stile e la sensibilità poetica. Il nostro scrittore si fa vedere come un letterato, un artista e un poeta e soprattutto un mistico che mediante le lettere intime e spirituali cerca l'aiuto ed i consigli dei direttori spirituali.

\subsection{Padre Benedetto ${ }^{34}$, il destinatario delle lettere}

Prima di proseguire a conoscere dell'Epistolario I di padre Pio è necessario tracciare una breve presentazione di padre Benedetto da San Marco in Lamis, che ha avuto molta importanza e svolto un ruolo delicato, qualche volta quasi determinante, in alcune vicende della vita del mistico di Pietrelcina. Padre Benedetto Nardella è nato a San Marco in Lamis il 16 marzo 1872. All'età di diciotto anni entrò nell'Ordine dei cappuccini vestendo l'abito religioso nel convento di Morcone, alcuni anni prima di padre Pio. Ordinato sacerdote nel 1898 si dedicò con passione all'insegnamento e all'educazione dei giovani. Aveva una preparazione non comune in filosofia e in teologia, ma coltivava anche la poesia e la letteratura. Eccelleva nell'arte dello scrivere sia in prosa sia in poesia; aveva un gusto naturale del bello e si dilettava di pittura e di scultura. Predicava con facilità di parola ed eleganza di stile. Possedeva il dono particolare della direzione spirituale delle anime. È stato nell'Ordine dei cappuccini superiore provinciale per 11 anni (dal febbraio 1908 al luglio del 1919) a Foggia, visitatore apostolico di altre province, delegato per capitoli generali, vicedirettore e direttore spirituale del Collegio Internazionale San Lorenzo da Brindisi a Roma. Ha conosciuto padre Pio il 25 aprile 1903, in visita a Morcone e l'ha avuto come studente nel convento di San Marco la Catola durante l'anno scolastico 1905-1906. E' stato scelto come direttore spirituale da padre Pio stesso nel 1905 e lo è rimasto fino al 1922, quando ha dovuto troncare qualsiasi rapporto sia verbale sia epistolare

34 Per altre notizie su padre Benedetto. Cf. Analecta Ord. Fr. Min. Cap., 58 (1942) p. 127s; In morte del M.R.P. Benedetto da S. Marco in Lamis, "L'Amico del Terziario", 15-16 (1941-1942) pp. 121136; Cipriano da Serracapriola, Necrologia dei Frati Minori Cappuccini della provincia monastica di Foggia, Foggia, 1969, p. 457; R. Fabiano, Padre Benedetto Nardella a S. Severo, "Studi su Padre Pio", 5/2 (maggio-agosto 2004), pp. 193-205. 
con il suo discepolo, per ordini superiori del Sant'Offizio. È morto a San Severo il 22 luglio 1942.

Le loro relazioni diventano frequenti dopo che padre Benedetto è stato eletto provinciale ed ha iniziato ad occuparsi della situazione di padre Pio, costretto a vivere fuori convento. Il provinciale continua a fare la direzione spirituale, cominciata nel 1905. Alla direzione iniziale e quasi esclusiva di padre Benedetto si aggiunge, in seguito, quella di padre Agostino. Per circostanze più favorevoli di tempo e di luogo è proprio padre Agostino ${ }^{35}$ che occupa il primo posto per un periodo. La direzione diventa "collegiale". Però l'intervento di padre Benedetto rimane sempre più autorevole e decisivo; a lui sono indirizzati personalmente i resoconti di coscienza più importanti e più minuziosi. I rapporti di padre Pio con i direttori spirituali sono improntati ad uno spirito di fede incrollabile. La loro voce per lui è la voce di Dio. I direttori, senza venir meno al loro compito di guide e di maestri, sentono l'influsso trascendente del loro diretto e, a loro volta, diventano suoi discepoli. Non per iniziativa personale ma dietro richiesta esplicita e ripetuto intervento, il religioso assume le parti di maestro e di direttore dei suoi direttori. Il nuovo, delicato ed imbarazzante incarico inizia quasi contemporaneamente, verso il 1915, rispetto ai due direttori.

L'Epistolario I dimostra il modo e la frequenza dei rapporti intercorsi tra i due religiosi. Le lettere di padre Benedetto da San Marco in Lamis, letterariamente sono elaborate bene ${ }^{36}$. Padre Pio era consapevole che il Signore voleva servirsi di lui per svegliargli i suoi divini disegni e il modo concreto di attuarli ed anche per confermarlo sia sulla realtà dei fenomeni sia sul modo di comportarsi nei momenti sofferenti della via verso la cima della perfezione. La scelta di lui, come direttore, era la volontà di Gesù, come afferma nel contenuto della lettera

35 Padre Agostino è nato a San Marco in Lamis il 9 gennaio 1880. Il 19 agosto 1897 entrò nell'Ordine dei cappuccini. Elessero più volte superiore conventuale, per 24 anni consigliere del superiore della provincia di Foggia, per 9 anni ministro provinciale la stessa provincia. Durante i quasi sette anni di permanenza di Padre Pio in famiglia a Pietrelcina, gli fu "angelo consolatore", sia attraverso le lettere di direzione e sia con frequenti visite personali. Poi, fu collaboratore di padre Benedetto nella direzione spirituale del Stigmatico.

${ }_{36}$ Cf. Melchiorre da Pobladura e Alessandro da Ripabottoni, Introduzione, in Epist. I, 52. 
del 4 giugno del 1918, in cui padre Pio scrive: "Rammentatevi che a voi il Signore mi affidò per guida, conforto e salvezza" ${ }^{37}$. Padre Benedetto, guida spirituale del religioso, ci tiene ad essere regolarmente informato dei suoi bisogni e dei progressi, come pure delle esigenze di Dio a suo riguardo. Nella sua direzione è "esigente, alquanto autoritario ed alle volte sembra poco espansivo e quasi staccato, senza indulgere al sentimentalismo" ${ }^{38}$. Testimone convinto dei doni mistici del suo discepolo, li mantenne segreti finché gli fu possibile, e non fu affatto credulo nell'accettarli. Nonostante certe passeggere difficoltà e incomprensioni, lui godette sempre la stima, la venerazione e la incondizionata adesione di padre Pio. Fu il suo vero maestro nelle vie dello spirito e il suo autorevole direttore. Padre Benedetto da San Marco in Lamis, mostrato come autorevole ministro provinciale, esperto nella direzione delle anime, acquista presso il suo confratello di Pietrelcina un tale prestigio da diventare la sua guida, il suo maestro e perciò il destinatario della sua corrispondenza spirituale.

\subsection{Epistolario I - la caratteristica generale}

Voglio adesso presentare il breve studio della corrispondenza letteraria di padre Pio con padre Benedetto da San Marco in Lamis, esaminando le sue lettere sotto i seguenti aspetti: l'origine, l'autenticità del contenuto, la frequenza e la lunghezza delle sue lettere. La lettura delle lettere di padre Pio, in modo chiaro, ci mostra l'origine e i motivi per cui sono state scritte. Non giungendo a manifestare per iscritto la sua vita intima e i suoi rapporti con la divinità "né da capriccio né dalla vana compiacenza, né da qualunque altro motivo umano", se scrisse fu solo per "compiere la volontà di Dio e per soddisfare alle impellenti necessità dello spirito, assetato di luce e di conforto"39.

Ha un valore programmatico ciò che egli scriveva a padre Benedetto già nell'ottobre del 1910: "Mi scriva, poiché mi fanno tanto bene i suoi

\footnotetext{
$37 \quad$ Epist. I, 1029.

38 Cf. Melchiorre da Pobladura e Alessandro da Ripabottoni, Introduzione, in Epist. I, 53.

${ }_{39}$ Cf. Melchiorre da Pobladura e Alessandro da Ripabottoni, Introduzione, in Epist. I, 58.
} 
consigli e mi dica ancora ciò che Dio vuole da questa ingrata creatura" ${ }^{40}$. E pochi giorni più tardi "questa ingrata creatura", come egli stesso si chiamava, aggiunge: "Se non le dispiace mi scriva, poiché mi fanno tanto bene le sue lettere" ${ }^{41}$. La direzione spirituale di padre Benedetto attraverso la corrispondenza, come assicura padre Pio, gli "fa tanto bene" e gli rivela la volontà di Dio. Desidera lettere lunghe dal suo direttore spirituale, ma egli è piuttosto breve nei suoi resoconti di coscienza, a meno che non fosse stato espressamente richiesto il contrario. Perciò padre Benedetto per stimolarlo, e come reazione al desiderio di ricevere lettere lunghe, quasi come una minaccia, gli scrive "di non essere breve solo quando tu sarai lungo" ${ }^{42}$. Dal conto suo anche padre Benedetto desidera che il suo discepolo gli scriva più spesso e più lungo e il motivo di tale esigenza era duplice: "da una parte, vuole essere informato tempestivamente dello stato dell'anima per rendere più efficace la direzione; e dall'altra, anche egli ha bisogno dei suoi consigli e orientamenti" ${ }^{43}$.

Possiamo anche confermare l'autenticità dell'Epistolario I. Le lettere "sia quelle di padre Pio che quelle dei suoi direttori sono state trascritte dagli autografi, quindi ogni sospetto a riguardo sarebbe infondato" 44 . Al religioso non competeva conservare le lettere spedite ai direttori, i quali si premuravano di farlo di propria iniziativa. Invece egli conservava le lettere dei suoi direttori per duplice motivo: "per il vantaggio spirituale che ricavava dalla lettura delle medesime e perché così gli era ordinato in virtù dell'obbedienza" ${ }^{45}$. Il testo autografo delle lettere si conserva attualmente nell'archivio dei Cappuccini di San Giovanni Rotondo. A questo criterio si aggiunge il criterio di valore interno del contenuto e della dottrina.

$40 \quad$ Epist. I, 200.

41 Epist. I, 203.

42 Epist. I, 1217.

43 Cf. Melchiorre da Pobladura e Alessandro da Ripabottoni, Introduzione, in Epist. I, 60; Cf. Epist. I, 1149; 1231; 1235.

${ }^{44}$ Cf. Melchiorre da Pobladura e Alessandro da Ripabottoni, Introduzione, in Epist. I, 64.

${ }_{45}$ Cf. Melchiorre da Pobladura e Alessandro da Ripabottoni, Introduzione, in Epist. I, 64. 
Adesso passiamo al contenuto dell'Epistolario I. Vogliamo accennare che l'argomento delle lettere è uno ed allo stesso tempo vario. E' uno, perché "le lettere non hanno altro scopo che di rendere un esatto resoconto ai direttori dello stato presente della sua anima, manifestando loro i segreti dello spirito per trovare sicurezza nel cammino, orientamenti nei dubbi e sostegno nelle perplessità" ma è altresì varia la tematica, perché "varie sono le fasi dell'attuazione dei disegni divini, varie manifestazioni della grazia, varie le difficoltà e le lotte della natura e le insidie di satana, varie le aspirazioni ed i bisogni dell'anima, varia la fenomenologia mistica, che punteggia l'itinerario dell'anima verso Dio"46. L'Epistolario I contiene le pagine traboccanti di dolore e amarezza, che traducono, in termini approssimativi, aspetti inediti o poco noti della "vita dolorosa del Calvario che conduce alle delizie dell'unione trasformante del Tabor" ${ }^{47}$.

In questa parte del paragrafo vogliamo descrivere il contenuto dell'Epistolario I, elencando le lettere in esso raccolte, sia quelle di padre Pio, sia quelle di padre Benedetto. Complessivamente l'Epistolario I. Corrispondenza con i direttori spirituali (1910-1922) riporta il testo autografo di 633 lettere, cioè 334 del padre Pio, 197 di padre Agostino e 102 di padre Benedetto. Il carteggio conta 162 lettere di padre Pio indirizzate a padre Benedetto e 172 lettere indirizzate a padre Agostino. La corrispondenza epistolare dimostra il modo e la frequenza dei rapporti intercorsi tra padre Pio e padre Benedetto. Complessivamente abbiamo 264 lettere (162 di padre Pio e 102 di padre Benedetto). Le lettere di padre Pio sono giunte a noi, invece la corrispondenza di padre Benedetto è più lacunosa, come apparirà dalle note a piè di pagina ${ }^{48}$. Molto probabilmente le lettere mancanti furono distrutte, in un secondo tempo, dallo stesso autore, perché in esse aveva descritto il suo mondo interiore come quasi confessione.

46 Cf. Melchiorre da Pobladura e Alessandro da Ripabottoni, Introduzione, in Epist. I, 72.

47 Cf. Melchiorre da Pobladura e Alessandro da Ripabottoni, Introduzione, in Epist. I, 77.

48 Cf. Epist. I, in nota (1), 220; in nota (1), 229; in nota (1), 245; in nota (1), 308; in nota (2), 370 . 
La scala sull'asse degli anni di corrispondenza da 1 a 13, sia sul diagramma sia sul grafico, denoterà uno dopo l'altro gli anni dal 1910 al 1920.

Il paradigma annuale del carteggio è il seguente:

1910: 25. - padre Pio 16, padre Benedetto 9.

1911: 21. - padre Pio 14, padre Benedetto 7.

1912: 11. - padre Pio 8, padre Benedetto 3.

1913: 17. - padre Pio 10, padre Benedetto 7.

1914: 10. - padre Pio 7, padre Benedetto 3.

1915: 21. - padre Pio 12, padre Benedetto 9.

1916: 13. - padre Pio 8, padre Benedetto 5.

1917: 33. - padre Pio 20, padre Benedetto 13.

1918: 33. - padre Pio 21, padre Benedetto 12.

1919: 16. - padre Pio 10, padre Benedetto 6.

1920: 14. - padre Pio 7, padre Benedetto 7.

1921: 38. - padre Pio 22, padre Benedetto 16.

1922: 12. - padre Pio 7, padre Benedetto 5.

Grafico annuale del carteggio tra padre Pio e padre Benedetto negli anni 1910-1922

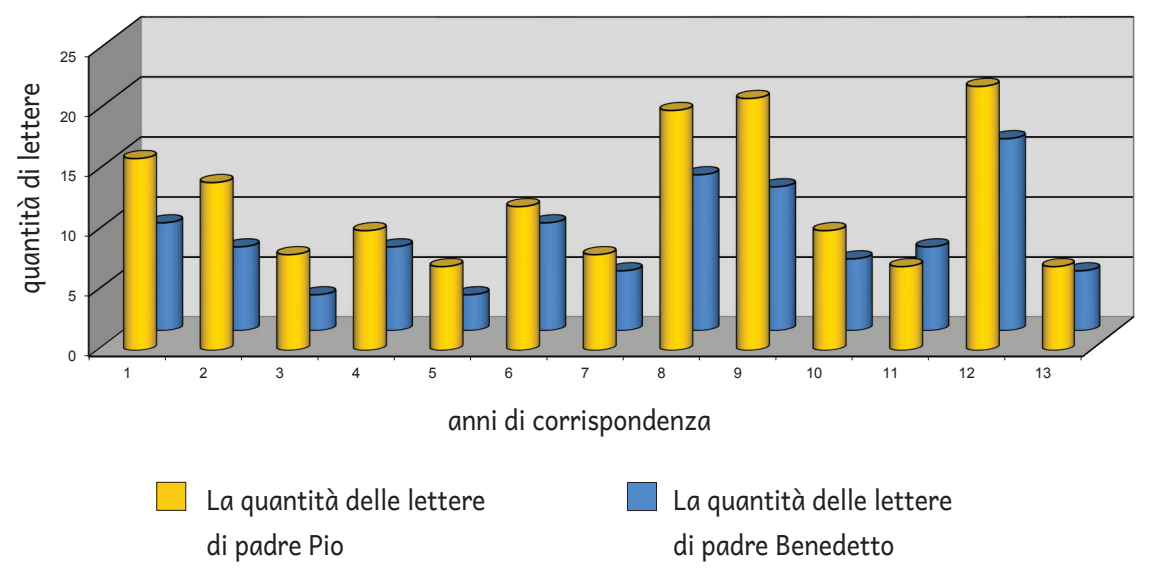


Grafico annuale del carteggio tra padre Pio e padre Benedetto negli anni 1910-1922

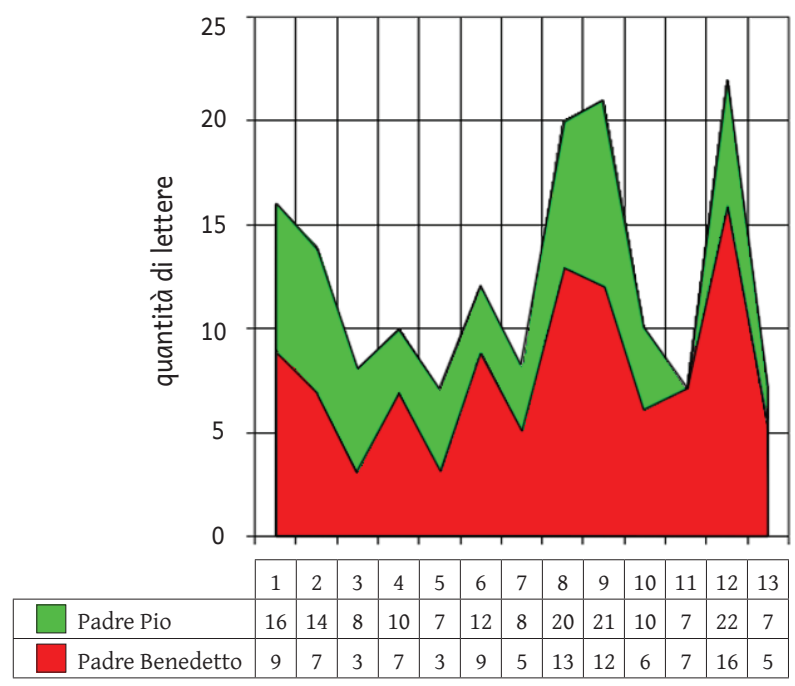

anni di corrispondenza

Infine, ci proponiamo di ricordare e analizzare alcuni fattori soltanto, che spiegano e giustificano la condotta di padre Pio circa la frequenza e la lunghezza delle sue lettere a padre Benedetto. Spesso il suo abituale stato cagionevole di salute non gli permette di sbrigare la corrispondenza tempestivamente ed il primo a soffrirne è proprio lui. Non mancano sgorbi di penna specialmente quando scrive tormentato da altissime febbri, da emicranie lancinanti o con la vista indebolita. Si aggiungano le suggestioni e l'intervento di satana per ostacolare la regolare corrispondenza con il suo direttore spirituale. La frequenza e la lunghezza delle lettere è condizionata anche dalle assillanti occupazioni di ministero (si vedano sul diagramma e sul grafico gli anni 1919-1920), soprattutto a partire dal 1919, quando le folle cominciavano a riversarsi al convento di San Giovanni Rotondo.

Il dirigere per iscritto, da parte di padre Benedetto, non era così intenso e frequente, invece, il suo discepolo scrive tanto di più (Cf. diagramma e grafico) e lo sollecita a farlo presto e lungamente. Padre Benedetto alle 
volte fa il sordo e senza volerlo rende più difficile il cammino. Sia il diagramma che il grafico mostrano la frequenza del carteggio tra padre Pio e padre Benedetto negli anni 1910-1922. La ampiezza sul diagramma riporta agli importanti fatti di natura religioso-disciplinare e di natura spirituale nella vita di padre Pio, sottolineando l'intensificata corrispondenza: negli anni 1910-1911, per la difficoltà di ricevere la dispensa per l'ordinazione sacerdotale e la sua prolungata permanenza fuori dal convento; negli anni 1917-1918, a causa del servizio militare a Napoli e dell'impressione delle stimmate e nel 1921, per l'intensità della caligine della notte oscura e della direzione spirituale, che fa per padre Benedetto.

\section{Il fenomeno di sofferenza}

L'Epistolario I è una letteratura sulla sofferenza che offre un itinerario spirituale, indicando all'uomo che va a cercare Dio per capire e vivere la sofferenza salvatrice il cammino da seguire. Le lettere, tendono a parlare, con notevole frequenza, dei dolori, delle sofferenze e dei tormenti intimi di padre Pio. La sofferenza è "un'esperienza umana fondamentale, che interpella vivamente l'uomo ai vari piani della conoscenza: psicologico, filosofico, religioso e le forme e i gradi di questa esperienza si presentano oggi molto numerosi e variati a tutti i livelli: spirituale, psichico, somatico" ${ }^{49}$. Noi intendiamo la parola "sofferenza" secondo il concetto generale, dividendolo in due gruppi: il dolore fisico e il dolore spirituale, seguendo lo stesso schema di Antonio Queralt ${ }^{50}$, specialista e professore di ascetica e mistica presso la Pontificia Università Gregoriana. Il male, come presenza e esistenza della sofferenza sia fisica che spirituale quale conseguenza del peccato, produce tanti problemi dal punto di vista della filosofia e della teologia. Il nostro studio non pretende di approfondire questa tematica, ma analizza il fenomeno del-

49 F. Ruiz, Sofferenza, [in:] Dizionario enciclopedico di spiritualità, vol. III, a cura di E. Ancili, Roma 1990, pp. 2334-2335.

50 Cf. A. Queralt, El «dolor»: su valor cristologico. Estudio teologico-spiritual del autotestimonio del P. Pio de Pietrelcina, O.F.M.Cap., in QS, 127. 
la sofferenza, come nozione generale, dal punto di vista degli effetti che produce. Vogliamo, per quanto è possibile, fino ad un certo grado, usare come sinonimi le parole "sofferenza" e "dolore". La sofferenza fisica si verifica quando in qualsiasi modo "duole il corpo", mentre la sofferenza spirituale è "dolore dell'anima”.

\subsection{I dolori fisici}

La sofferenza implica il sentimento di un dolore, il male che apporta al soggetto, è un'esperienza forte e diretta come sensazione prevalente di tutta l'esistenza in vasti settori. Noi vogliamo chiamare "dolori fisici" quei patimenti, sofferenze, tormenti, che per una causa immediata e materiale, si possono considerare localizzati in un organo o una parte del corpo vivo e trasmessi attraverso il sistema nervoso. Attendendo alla testimonianza di padre Pio possiamo raggruppare questi dolori in classi. La prima comprenderà tutti i dolori che sono dovuti a cause naturali, infermità o disfunzione di qualche organo, producendo le diverse sofferenze e dando il patimento a chi lo soffre. Nella seconda raccoglieremo, attraverso le prove oculari, i dolori che sono stati attribuiti a un'ingerenza diabolica o altresì, a causa delle prove che si devono attribuire a un particolare intervento di Dio.

\section{La salute fisica}

Quasi tutti i mistici trovarono difficoltà non indifferenti riguardo alla costituzione e alla salute. La nostra breve esposizione vuole mostrare queste difficoltà, che accompagnarono padre Pio. Cominciamo dal raccogliere i dati sulla salute fisica del mistico da Pietrelcina. Nell'Epistolario I appare la cagionevolezza della salute e le conseguenze della sofferenza, che il religioso sopporta man mano. Tante volte, padre Pio descrive lo stato fisico della sua salute, dicendolo di solito, informa il suo direttore spirituale, padre Benedetto, allora il ministro provinciale, delle sue spese mediche ${ }^{51}$ :

${ }^{51}$ Cf. Epist. I, 178; 182; 184; 197; 451; 479; 483. 


\section{Błażej Strzechmiński OFMCap}

Mi dispenso di parlare della mia salute, poiché le continue infermità, che non vogliono lasciarmi, anziché diminuire la fiducia nella mia madre celeste e nel suo figliuol Gesù $[. . .]^{52}$; Vari giorni li ho passati più male del solito colla salute ${ }^{53}$.

Alcune lettere parlano più concretamente della sua salute, come questa del 20 dicembre 1913 a padre Benedetto, nella quale appare il suo cattivo stato di salute e la giustificazione per la sua permanenza in famiglia:

Voi che mi conoscete a fondo ed a cui tante volte ho aperto il cuore, voi potete immaginare con quanta gioia volerei in convento; ma poiché la mia malattia si va sempre accentuando e che a stento mi trascino, sarei di peso e d'ingombro alla comunità, senza apportare nessuno aiuto ed affrettando al mia fine ${ }^{54}$.

Per padre Pio il fenomeno rimane alquanto misterioso, ma egli non dubita mai dell'intervento divino riguardo all'origine a allo sviluppo della malattia, i cui laceranti effetti solo lui conosce:

Io sono convintissimo, dietro che ella me ne ha assicurato, che la malattia, essendo una speciale permissione di Dio e quindi non esservi bisogno di medici ${ }^{55}$.

Il religioso, che sente tanti dolori fisici, mai si fa illusione di guarigione, anche se per esigenze umane contingenti o per motivo d'obbedienza alle volte si sottomette a certe cure mediche:

L'idea della guarigione, dietro tutte queste tempeste che l'Altissimo da su mi manda, sembrami un sogno, anzi una parola priva di senso ${ }^{56}$; Io ne ignoro la causa di ciò ed in silenzio adoro e bacio la mano di colui, che mi percuote ${ }^{57}$.

La salute di padre Pio non solo non fu florida, ma andò sempre peggiorando con gli anni.

\footnotetext{
52 Epist. I, 185.

53 Epist. I, 199.

54 Epist. I, 444.

55 Epist. I, 241.

56 Epist. I, 180.

57 Epist. I, 182.
} 


\section{Le malattie}

La sintomatologia, anche se non in termini tecnici, è variamente descritta nell'epistolario e presenta un quadro clinico assai complesso dei malanni che affliggevano il fragile organismo: febbri altissime ed assai frequenti, affezioni polmonari, strazianti dolori al petto, indebolimento della vista, reumatismi paralizzanti, intensi sudori, vomito e emicranie fortissime ${ }^{58}$. Le malattie di padre Pio sono state fatte oggetto di attento studio da parte del dottore Michele Capuano, il medico di San Giovanni Rotondo. Nel seguente capitolo vogliamo seguire il suo studio, confrontarlo con le lettere indirizzate a padre Benedetto da San Marco in Lamis. La diagnosi di "bronco-alveolite nel primo stadio"59 all'apice sinistro, fatta dai medici a padre Pio nel 1909, era la prima opinione medica ufficiale, che trattava sulla sua misteriosa malattia. Nell'ospedale a Napoli, nel 1915, la visita collegiale della commissione medica ancora una volta riconosce la malattia di padre Pio, come infiltrazione polmonare ${ }^{60}$. Nel 1917, la solita diagnosi d'infiltrazione polmonare gli viene confermata altre sei volte presso l'Ospedale napoletano della Trinità, e due volte all'Università nella Clinica Medica, dov'è andato in osservazione, insieme a catarro bronchiale e mali minori ${ }^{61}$. Il religioso, nella lettera a padre Benedetto, descrive il suo stato fisico con le parole drammatiche: "Tutto il mio corpo, è un corpo patologico. Catarro bronchiale diffuso, aspetto ischeletrito, nutrizione meschina e tutto il resto" ${ }^{2}$. Padre Pio contrae più volte anche infezioni broncopolmonari ${ }^{63}$. Forse per talune di queste broncopolmoniti rimane il dubbio che finirono ignorate, perché confuse con attacchi acuti d'influenza vera, o con manifestazioni da raffreddore più forti del solito.

${ }_{58}$ Melchiorre da Pobladura e Alessandro da Ripabottoni, Introduzione, in Epist. I, 22; Cf. G. Saldutto, Un tormentato settennio (1918-1925) nella vita di Padre Pio da Pietrelcina, Roma 1974, p. 91.

59 M. Capuano, Padre Pio uomo di dolore (Le malattie di padre Pio viste da un medico), [in:] QS, 17.

60 Cf. Epist. I, 699.

${ }_{61}$ Cf. M. Capuano, Padre Pio uomo di dolore (Le malattie di padre Pio viste da un medico), [in:] QS, 20; Cf. Epist. I, 853; 931.

62 Epist. I, 937.

63 Cf. M. Capuano, Padre Pio uomo di dolore (Le malattie di padre Pio viste da un medico), [in:] QS, 30. 
Dall'epistolario indaghiamo che nei primi mesi del 1910 il vomito s'aggiunse ai sintomi broncopolmonari, ma in una maniera così violenta, da indurlo a disperare della guarigione, o paventandogli perfino la morte:

Lo stomaco, grazie al cielo, è quasi da Natale [l'anno 1909] che non rigetta più nulla, mentre per lo innanzi riteneva appena la sola semplice acqua. Anche le forze me le sento alquanto ritornate, tanto da poter camminare per un po' senza tanto incomodo. Ma ciò che non vuol lasciami si è la febbre, che quasi tutti i giorni, verso sera, viene a farmi visita, seguita da copiosi sudori. La tosse poi ed i dolori del torace e della schiena sono quelli che più mi martirizzano continuamente ${ }^{64}$.

Nel luglio del 1910, il dolore acuto al polmone sinistro è così forte, che egli non può parlare ${ }^{65}$. In autunno ritornano la tosse e dei dolori al torace. La tosse è talmente forte ed insistente "che poco manca da non spezzarsi il petto" ${ }^{66}$, scrive il religioso ammalato a padre Benedetto. Padre Pio, in questo tempo, ha la febbre tutti i giorni fino alla metà di aprile dello stesso anno ${ }^{67}$ e aumentano nei mesi seguenti anche le fitte al tora$\mathrm{ce}^{68}$. La febbre di padre Pio è diventata cronica ed è chiamata dai medici la febbre a permanenza, perché non accenna a "decadere" ${ }^{69}$. Questa diagnosi è motivo per l'esonero definitivamente dal servizio militare. La lettera da Foggia del 13 agosto 1916 indirizzata a padre Benedetto accenna alle "febbri malariche", ed è una diagnosi non fatta dai medici, ma da padre Pio stesso, in base al freddo, cioè ai brividi, che gli venivano di sera ${ }^{70}$. Probabilmente quelle erano febbri reumatiche. Nelle sue lettere parla tante volta di vomito, e di uno stomaco che s'era ridotto a non ritenere più niente $^{71}$. Il 19 settembre del 1917, continua a scrivere a padre Benedetto, che: "Lo stomaco si va sempre più ostinando a non reggere cibo alcuno e giorni fa ebbi anche un'emottisi di sangue"72.

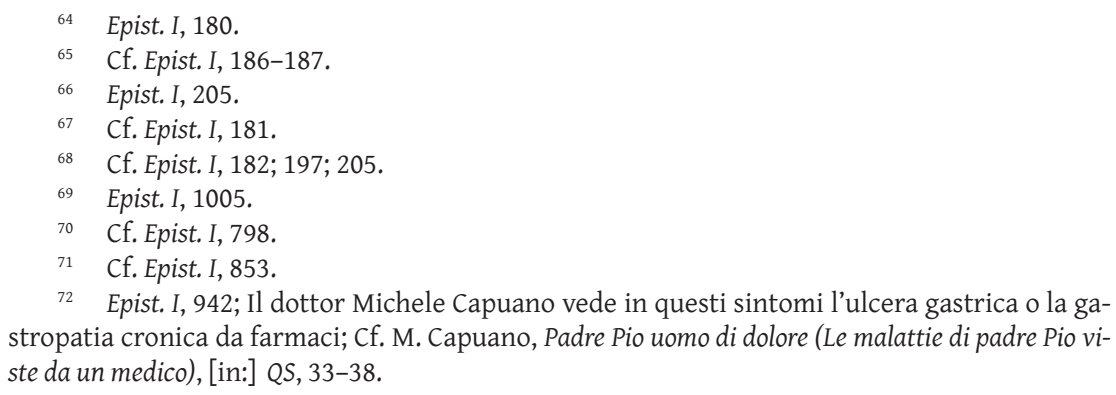

72 Epist. I, 942; Il dottor Michele Capuano vede in questi sintomi l'ulcera gastrica o la gastropatia cronica da farmaci; Cf. M. Capuano, Padre Pio uomo di dolore (Le malattie di padre Pio viste da un medico), [in:] QS, 33-38. 
Il religioso cappuccino, nel 1912, si lamenta del dolore "acutissimo" al braccio destro ${ }^{73}$, e già nel 1914 cominciano fin da gennaio i reumatismi. Padre Pio confessa nella lettera indirizzata a padre Benedetto: "Da vari giorni in qua, oltre alle solite ed ordinarie molestie, alle quali vado soggetto colla mia salute, si è aggiunta quella di sentirmi tutto il corpo reumatizzato"74. Questa malattia, in aggiunta alle fitte toraciche preesistenti, gettano il religioso in grande e generale prostrazio$\mathrm{ne}^{75}$, fino ad impedirgli quasi di scrivere e di celebrare la messa. Il 1915 è l'anno delle fitte e dei dolori alla testa che continuano, fino a spaccargli la fronte, e a sottrarlo, a "qualsiasi applicazione"76. La cefalea, che era la manifestazione più inquietante, passava dal forte al fortissimo e al violentissimo, trasformando la sua vita in un crudele martirio $^{77}$. Le emicranie di non minore violenza erano spesso così tremende, da diventare insoffribili ${ }^{78}$.

La "cecità"79 di padre Pio è più volte ricordata nell'epistolario ed è probabile che cominciasse ad averla nel 1911. Egli non vede le righe della carta da lettera e in pratica va storto quando scrive, tuttavia padre Benedetto gli raccomanda di scrivere lo stesso, per non privarlo della consolazione di ricevere sue notizie ${ }^{80}$. I disturbi agli occhi di padre Pio non migliorano l'anno seguente, e nel gennaio del 1912, la deficienza visiva è la stessa ${ }^{81}$. Questo problema si trascina a lungo, tra alti e bassi. Nel 1915 non è ancora scomparso, tanto da indurlo praticamente a sostituire l'Ufficio con la recita del rosa-

73 Cf. Epist. I, 297; Il dottor Michele Capuano, nel dolore al braccio destro di padre Pio, afferma la sindrome spalla-mano dello Steinbrocker; Cf. M. Capuano, Padre Pio uomo di dolore (Le malattie di padre Pio viste da un medico), in QS, 45.

74 Epist. I, 451.

75 Epist. I, 483.

76 Epist. I, 583.

77 Cf. Epist. I, 217; 383; 797.

78 Cf. Epist. I, 591.

79 Probabile, questa specie di cecità di padre Pio è una rara forma di "cecità transitoria" oppure come vogliono chiamare inglesi Shafer e Hollanders: "blindness episodic benign" (cecità episodica benigna); Cf. M. Capuano, Padre Pio uomo di dolore (Le malattie di padre Pio viste da un medico), [in:] QS, 61.

${ }^{80}$ Cf. Epist. I, 248

${ }^{81}$ Cf. Epist. I, 250. 
rio e dopo più di tre anni, la vista, come per miracolo, gli si schiarisce a tal punto, da fargli desiderare di tornare al breviario ${ }^{82}$. C'è poi un silenzio di quattordici anni ${ }^{83}$, durante i quali non si hanno più notizie relative alla vista.

I sintomi accennati nelle lettere affermano che il fatto patologico è evidente. Vale la pena annotare che "nessun medico fu mai in grado di offrire una diagnosi soddisfacente e convincente e nessuno specialista riuscì a spiegare scientificamente la presenza latente e la causa proporzionata dei sintomi più sconcertanti, con la loro improvvisa ed altrettanto sconcertante scomparsa" ${ }^{84}$. La scienza si dichiarava impotente, anzi vinta e la terapia applicata inutile, perciò padre Pio scrive:

Il medico mi ha suggerito queste parole: non ho che farti ${ }^{85}$ Le medicine che ho prese, come se l'avessi gittate in un pozzo ${ }^{86}$; Presentemente poi dietro le moltissime medicine che ho preso e che sto prendendo, massime di iniezioni ipodermiche, che ne ho fatto tante che non ho quasi più dove bucare ${ }^{87}$.

Niente esaltazioni dei suoi dolori fisici, come qualcuno ha tentato d'insinuare, attribuendogli uno stato generico d'iperestesia viscerale e muscolo-cutanea, condizionato a sua volta da una psiche malata, al contrario, padre Pio minimizzava il dolore fisico, nascondendolo, perfino ai medici, e non apprezzava le cure affatto, ma nello spirito di ubbidienza e di umiltà era sottomesso al consiglio dei medici ${ }^{88}$. La verità è che padre Pio amava il dolore, che lo sopportava con rassegnazione, anzi con gioia, minimizzandolo quasi sempre senza dargli importanza, pur di sfuggire, come diceva, "alla cecità dell'anima"89.

82 Cf. Epist. I, 523; Cf. M. Capuano, Padre Pio uomo di dolore (Le malattie di padre Pio viste da un medico), in QS, 59.

${ }_{83}$ Cf. M. Capuano, Padre Pio uomo di dolore (Le malattie di padre Pio viste da un medico), [in:] QS, 59 .

${ }_{84}$ Melchiorre da Pobladura e Alessandro da Ripabottoni, Introduzione, [in:] Epist. I, 21.

85 Epist. I, 224.

86 Epist. I, 225.

87 Epist. I, 230.

88 Cf. M. Capuano, Padre Pio uomo di dolore (Le malattie di padre Pio viste da un medico), [in:] QS, 63.

${ }_{89}$ Cf. Epist. I, 246. 


\subsection{I dolori fisici "soprannaturali"}

Dopo aver presentato i dolori fisici naturali, sullo sfondo delle malattie di padre Pio, voglio delineare il quadro delle sofferenze fisiche "soprannaturali", seguendo lo stesso schema di Antonio Queralt ${ }^{90}$. Proviamo a dividerle in due classi opposte tra loro: di origine diabolica e di origine divina. Le prime le denominiamo "vessazioni" e le seguenti "grazie" speciali. Queste classi le consideriamo dal punto di vista del dolore fisico.

\section{Le vessazioni diaboliche}

L'intervento demoniaco nella vita degli uomini e specialmente dei santi è ben documentato nella letteratura degli autori spirituali e nella Bibbia. La nostra presentazione ci vuole mostrare gli interventi diabolici soprattutto sotto l'aspetto del dolore, indicando quelli che producono principalmente dolori fisici, come il combattimento. Gli interventi del diavolo che portano sofferenza morale, come tentazione e altre varie forme di suggerimenti che allontanano da volontà di Dio, li presenteremo nel capitolo riguardante alcune cause della sofferenza spirituale. Il riferimento ai testi scelti dell'Epistolario I, ci permetterà di seguire l'evoluzione della componente diabolica nell'esperienza di padre Pio e di mostrare la modalità dell'azione del diavolo. In questo paragrafo, voglio presentare i segni prolettici, come l'introduzione alla nostra tematica, ed i violenti attacchi diabolici alla persona di padre Pio.

I segni prolettici.L'esperienza mistica di padre Pio si caratterizza, tra l'altro, per un particolare accanimento diabolico contro la sua persona, il suo operato, il suo cammino spirituale, mediante la continua opera infestatrice e devastatrice del demonio, esplicatasi nei seguenti termini: dalle suggestioni alle apparizioni, dalle minacce alle percosse, dall'attacco diretto a quello indiretto - si pensi alle calunnie, agli strani ed improv-

90 Cf. A. Queralt, El «dolor»: su valor cristologico. Estudio teologico-spiritual del autotestimonio del P. Pio de Pietrelcina, O.F.M.Cap., [in:] QS, 134. 
visi impedimenti nell'apostolato e nel ministero della riconciliazione ${ }^{91}$. Sappiamo che le prime apparizioni diaboliche, a padre Pio, cominciarono all'età di cinque anni e si protrassero per quasi venti anni, prevalentemente in forme oscene ${ }^{92}$; tuttavia, padre Benedetto indica nell'età di circa quattro anni l'inizio delle vessazioni diaboliche: "il diavolo si presentava in figure raramente laide, spesso minacciose, orribili, spaventose. Era tormento, anche di notte non lo lasciavano dormire" ${ }^{\text {"93 }}$.

I Cenni autobiografici nell'Epistolario I contengono una lettera ${ }^{94}$, in cui padre Pio racconta di una visione avuta pochi giorni prima di entrare in convento. Il testo, che si fa prolettico e per certi aspetti programmatico, racchiude questi elementi: lo scontro personale, sproporzionato tra le due parti, senza esclusione di colpi, con un sorprendente risultato, cioè la vittoria del debole e la sconfitta del forte. La visione gli prospetta tutto l'arco della sua futura esistenza, vista come una continua ed accanita lotta contro il demonio. Vediamo ora l'intensità e gli effetti delle dolorose vessazioni ed i costanti, violenti e mirati attacchi diabolici sulla persona di padre Pio.

Il combattimento. Nelle lettere di padre Pio troviamo molteplici accenni e non poche descrizioni colorite delle lotte sostenute contro satana e i suoi alleati. I racconti più numerosi e più drammatici dei combattimenti con il diavolo sono trasmessi a padre Agostino, ma non mancano neanche nella corrispondenza con padre Benedetto, la quale sarà la base per le nostre considerazioni. L'Epistolario I ci suggerisce due periodi delle lotte: il primo è quello che va dal 1910 al 1916, e si caratterizza cogli assalti che colpiscono la sfera empirica del frate cappuccino mediante il mascheramento, prevalentemente, sotto mentite spoglie corporali, incrudendone i sensi; il secondo che va dal 1916 al 1922, vede il demonio impegnato nell'attaccare le facoltà dell'uomo, l'intellet-

${ }^{91}$ Cf. L. Lavecchia, L'itinerario di fede di Padre Pio da Pietrelcina nell'Epistolario, San Giovanni Rotondo 2004, pp. 72-73.

${ }_{22}$ Cf. Agostino da San Marco in Lamis, Diario, $2^{\text {a }}$ ed., a cura di G. Di Flumeri, San Giovanni Rotondo 1975, p. 58.

${ }_{93}$ Cf. Fernando da Riese San Pio X, Padre Pio da Pietrelcina, crocifisso senza croce, $3^{\text {a }}$ ed., San Giovanni Rotondo 1987, p. 44.

94 Cf. Padre Pio Da Pietrelcina, Cenni autobiografici di Padre Pio, [in:] Epist. I, 1280-1281. 
to, la volontà, per giungere alle virtù teologali ed inibirle ${ }^{95}$. La frequente presenza diabolica nella vita del religioso è tale da assumere addirittura i toni ironici, sarcastici nei riferimenti al demonio, come dimostrano i diversi epiteti, pur sempre dispregiativi, con cui padre Pio lo indica ${ }^{96}$. Il primo riferimento sul demonio lo incontriamo nella lettera datata 6 luglio 1910, padre Pio scrive:

Una nuova guerra mi va muovendo il principe delle tenebre. Questi essendo rimasto vinto da una parte per averla ubbidito, confesso certamente non esser mio merito, un'altra battaglia non meno accanita dell'altra mi va facendo ${ }^{97}$.

In questa prima relazione il religioso rivela che l'azione del demonio non gli è una novità, ma è il suo nuovo accanito attacco. L'obbedienza diventa per lui una delle armi vincenti contro le insidie diaboliche.

Gli attacchi del demonio sono costanti. La costanza della presenza diabolica è motivata dalle finalità che il demonio vuole perseguire nella vita di padre Pio per strapparlo dall'amicizia con Dio per sterminar$10^{98}$. Spesso ritorna nelle lettere l'angoscia causata dal persistere dei costanti attacchi diabolici:

Anche durante le ore del riposo il demonio non lascia di affliggermi l'anima in vari modi"9; [...] il nemico della nostra salute è talmente arrabbiato che non mi lascia quasi un momento in pace, guerreggiandomi in vari modi ${ }^{100}$;

Ho assiduo satana preso di me colle sue vivaci suggestioni. [...] perché me lo veggo sempre dintorno e sempre ritorna all'assalto ${ }^{101}$.

95 Cf. Melchiorre da Pobladura e Alessandro da Ripabottoni, Introduzione, [in:] Epist. I, 128129; Questo secondo periodo delle lotte, come la crudele tentazione diabolica, sarà presentato nel paragrafo Le tentazioni.

96 Ecco la lunga sfilza degli epiteti usati da padre Pio nell'Epistolario I per nominare il demonio: baffettone, baffone, barbablù, birbaccione, infelice, spirito maligno, cosaccio, brutto cosaccio, brutto animalaccio, triste cosaccio, brutti ceffoni, impuri spiriti, quei disgraziati, malvagio spirito, bestiaccia, maledetta bestia, apostata infame, impuri apostati, facce patibolari, fiere che ruggiscono, insidiatore maligno, principe delle tenebre; Cf. Melchiorre da Pobladura e Alessandro da Ripabottoni, Introduzione, [in:] Epist. I, 129.

97 Epist. I, 187.

98 Cf. Epist. I, 219.

99 Epist. I, 203.

100 Epist. I, 205-206.

101 Epist. I, 1074. 
Dalla lettera del gennaio 1912 padre Pio rivela che alle antiche metodologie demoniache si sono aggiunte le percosse ${ }^{102}$. Il dolore fisico nasce dagli attacchi violenti del demonio, martellanti, intensi ed efficaci. La violenza fisica si manifesta attraverso i segni tangibili sia nei trattamenti crudeli del demonio, sia nelle ferite e nei lividi rilasciati sul corpo della vittima. Nella lettera datata 8 aprile 1913, padre Pio descrive al suo direttore spirituale uno di questi attacchi diabolici, da cui traspare la drammaticità dei fatti:

Quei cosacci non cessano di percuotermi e di sbalzarmi alle volte anche dal letto, giungendo fino a togliermi la camicia e percuotermi in tale stato. Ma ormai non mi fanno quasi più timore ${ }^{103}$.

Un'altra lettera racconta del furore di satana, che lo attacca nel giorno e nella notte:

Ancora una volta il Signore mi ha esposto al furore di satana. I di lui assalti sono violenti ed assidui [...]. Mi assale di giorno in tutte le ore; mi amareggia il sonno nelle ore della notte $\mathrm{e}^{104}$.

Da tanto tempo il religioso è bastonato dal demonio e suoi alleati, coloro che raddoppiano i propri sforzi per distruggerlo, perciò confessa: "mi sento più abbattuto nel corpo"105. Il 15 aprile 1915 sostiene, che è troppo debole in confronto della potente forza del demonio: "Veramente vi sono dei momenti, e questi non sono troppo rari, nei quali mi sento come schiacciato sotto la potente forza di questo triste cosaccio"106.

Alfine vogliamo presentare una lettera, questa volta indirizzata a padre Agostino, per sottolineare la violenza più furibonda del diavolo, diretta sulla persona di padre Pio:

Ed allorché videro andare in fumo i loro [cosacci] sforzi, mi si avventarono addosso, mi gittarono a terra, e mi bussarono forte, buttando per aria guanciali, libri, sedie, emettendo in pari tempo gridi disperati e pronunziando parole estremamente sporche ${ }^{107}$.

\footnotetext{
102 Cf. Epist. I, 252.

103 Epist. I, 353.

104 Epist. I, 966-967.

105 Epist. I, 325.

106 Epist. I, 562-563.

107 Epist. I, 330.
} 
Nell'Epistolario I si fa menzione diretta all'aspetto fisico del conflitto, colorando, così, di ulteriore drammaticità lo sfondo del duello. Il demonio fa uso della violenza fisica attraverso le vessazioni e gli attacchi "ammaccando" realmente il corpo di padre Pio. La presenza costante dei "cosacci" nella vita quotidiana del religioso aggiunge, dunque, il nuovo elemento: il dolore fisico.

\subsection{La sofferenza fisica a causa di "grazie"speciali}

Altro tipo di dolore ci sono le sofferenze fisiche a causa delle "grazie" speciali. Questa particolare sofferenza sarà divisa in tre fattori distinti: la transverberazione del fianco, la transverberazione del cuore e le stimmate. Tra le diverse grazie, che riceve padre Pio durante la sua vita, c'è la grazia speciale della transverberazione. Questa piaga d'amore ${ }^{108}$, come il segno della conformità con il Cristo crocifisso, sarà esaminata nel presente paragrafo alla luce del dolore sperimentato. La teologia cristiana, nel corso dei tempi, ha cristallizzato il concetto della transverberazione intendendola come "un particolare fenomeno mistico, che consiste in una ferita prodotta nell'anima con una piaga fatta al costo o al cuore o in tutte due [...] l'elemento formale e determinante del fenomeno è l'amore di Dio" ${ }^{109}$. Padre Pio riceve da Dio tutte le due "grazie" speciali: la piaga al costato e la piaga al cuore. Ci interessa osservare come la transverberazione si è prodotta in padre Pio, seguendo il contenuto delle lettere scritte a padre Benedetto da San Marco in Lamis. Inizieremo presentando la prima specie della piaga d'amore, la piaga al costo.

108 Nell'itinerario mistico dell'Epistolari I, la transverberazione è stata classificata come la piaga dell'amore oppure "assalto del Serafino". Cf. Melchiorre da Pobladura e Alessandro da Ripabottoni, Introduzione, [in:] Epist. I, 148.

109 G. Di Flumeri, La transverberazione di padre Pio da Pietrelcina, San Giovanni Rotondo 1985, 12. Il padre Gerardo Di Flumeri nel suo libro presenta uno studio sulla transverberazione di padre Pio, in cui affronta la problematica alla luce dell'esperienza mistica di santa Teresa d'Avila ed il pensiero di san Giovanni della Croce, per evidenziare le attualizzazioni, le affinità e le differenze fenomenologiche esistenti. 
La transverberazione del costato

Il fenomeno della transverberazione del costato si verifica nel religioso nei giorni dal 5 al 7 agosto del 1918. Padre Pio fa un racconto al suo direttore spirituale nella lettera del 21 agosto 1918:

Io non valgo a dirvi ciò che avvenne in questo periodo di superlativo martirio. Me ne stavo confessando i nostri ragazzi la sera del cinque, quando tutto di un tratto fui riempito di un estremo terrore alla vista di un personaggio celeste che mi si presenta dinanzi all'occhio della intelligenza. Teneva in mano una specie di arnese, simile ad una lunghissima lamina di ferro con una punta bene affilata, e che sembrava da essa punta che uccise fuoco. Vedere tutto questo ed osservare detto personaggio scagliare con tutta violenza il suddetto arnese nell'anima, fu tutto una cosa sola. A stento emisi un lamento, mi sentivo morire. Dissi al ragazzo che si fosse ritirato, perché mi sentivo male e non sentivo più la forza di continuare. Questo martirio duro, senza interruzione, fino al mattino del giorno sette. Cosa io soffrii in questo periodo sì luttuoso io non so dirlo. Persino le viscere vedevo che venivano stappate e stiracchiate dietro di quell'arnese, ed il tutto era messo a ferro e fuoco. Da quel giorno in qua io sono stato ferito a morte. Sento nel più intimo dell'anima una ferita che è sempre aperta, che mi fa spasimare assiduamente ${ }^{110}$.

La contestualizzazione della transverberazione di padre Pio evidenzia due elementi particolari: "il primo attiene alla prova mistica delle notte oscura, che da tempo sta vivendo e va attualmente portandosi verso gli ultimi stadi; il secondo riguarda il momento puntuale in cui viene a determinarsi: durante il ministero della confessione ${ }^{111}$. Il religioso mistico descrive la transverberazione, attraverso i termini più realistici, come il "periodo di superlativo martirio" riempito di "un estremo terrore" dal motivo di "un personaggio celeste" cui scaglia nell'anima "con tutta violenza" la "lunghissima lamina di ferro", facendo la luttuosa sofferenza. La ferita prodotta è di natura spirituale, "nel più intimo dell'anima”, ma chiaramente visibile anche sul corpo, con manifestazione inconfutabili, come scrive il religioso nella lettera del 5 settembre dello stesso anno: "Io mi veggo sommerso in un oceano di fuoco, la ferita che mi venne riaperta sanguina e sanguina sempre. Dessa sola basterebbe a darmi mille

\footnotetext{
110 Epist. I, 1065.

111 L. Lavecchia, L'itinerario di fede di Padre Pio da Pietrelcina nell'Epistolario, 175.
} 
e più volte la morte"112. Il testo tratta di una dolorosa ${ }^{113}$ ferita fisica al costato ${ }^{114}$ che "sanguina e sanguina sempre", rimanendo aperta.

Altra lettera indirizzata a padre Benedetto, del 17 ottobre del 1918, ove si scopre il dolore fisico e materiale della ferita della transverberazione al costato ${ }^{115}$, come "le piaghe antiche", che non vogliono cicatrizzarsi. Padre Pio fa la domanda esistenziale:

Sarà necessario che io pronunzi il fiat nel mirare quel misterioso personaggio che mi impiagò tutto e non desiste dalla dura, aspra, acuta e penetrante operazione, e non dà tempo che venga a rimarginare le piaghe antiche, che già su di queste ne viene ad aprire delle nuove con infinito strazio della povera vittima? ${ }^{116}$.

\section{La risposta a siffatta domanda giunge presto. Il sofferente religio- so confessa: "In mezzo a tanto strazio sento la forza di pronunziare il}

112 Epist. I, 1072.

113 Padre Pio scrive nella lettera del 5 settembre 1918: L'eccesso del dolore che mi cagiona la ferita che semprè è aperta; Epist. I, 1073.

114 Esiste una documentazione anatomica ad opera del prof. Amigo Bignami, in vista ufficiale a padre Pio per conto del Sant'Offizio, nel giugno del 1919: "Nel torace a sinistra, tra la linea ascellare anteriore e la ascellare media, si osserva una figura di croce, la cui branca più lunga, disposta obliquamente va dalla $5^{\mathrm{a}}$ alla $9^{\mathrm{a}}$ costola, raggiungendo il brodo costale, mentre la branca trasversale è dalla metà circa più breve. La cute non è infiltrata in modo riconoscibile..., non sanguina durante l'esame". La relazione del Dr. Giorgio Festa, in seguito alla visita fatta il 15 luglio 1920, porta le informazioni del simile contenuto; Cf. G. Cruchon, Le stimmate di Padre Pio, in Convegno I, 119; 122-123; Il padre Gerardo Di Flumeri nel suo libro La transverberazione di padre Pio da Pietrelcina presenta l'opinione la quale spiega che dalla testimonianza dell'Epistolario I non sia possibile avere una risposta sicura e definitiva alle domande: 1 . La lettera del 21 agosto 1918 parla della ferita nel cuore o nel costato o in tutte due? 2. La lettera del 5 settembre 1918, seguendo il suo contesto, afferma il significato fisico o simbolico, metaforico della ferita che sanguina? Tuttavia, l'autore del libro sulla transverberazione di padre Pio, finalmente, vede in queste lettere l'inscrizione della transverberazione del costo, uguale come Antonio Queralt. Noi seguiamo la stessa opinione, fondandosi la testimonianza di padre Paolino da Casacalenda della piaga al costato, che "ha la forma quasi di una X da che si deduce che sono due le ferite" (Paolino da Casacalenda, Le mie memorie intorno a Padre Pio, a cura di G. Di Flumeri, San Giovanni Rotondo 1978, p. 173). Questa testimonianza fatta dopo la transverberazione e dopo la stimmatizzazione di padre Pio, suggerisce che c'è una seconda piaga al costato, che è stata aggiunta a quella già esistente dopo la transverberazione.

115 Probabilmente padre Pio nella sua lettera del 17 ottobre del 1918 scrive in plurale, "le piaghe antiche", perché non solamente era trafitto il suo costato ma anche il mistero personaggio stappava e stiracchiava le sue viscere.

116 Epist. I, 1090. 
doloroso fiat. Oh fiat!, quanto sei dolce ed amaro insieme"117! Il corpo del religioso partecipa nella dura, aspra e acuta sofferenza fisica, ma la stessa grazia speciale della piaga al costato manifesta all'esterno la sofferenza spirituale. Il dolore fisico e la sofferenza spirituale si penetrano reciprocamente.

\section{La transverberazione del cuore}

Alla transverberazione dell'anima con ferita al costato aggiunge la grazia della transverberazione dell'anima con ferita fisica al cuore ${ }^{118}$. Nell'Epistolario I troviamo una testimonianza su questo argomento, nella lettera del 20 dicembre 1918, scritta a padre Benedetto:

Da più giorni avverto in me una cosa simile ad una lamina di ferro che dalla parte bassa del cuore si estende sino a sotto la spalla destra in linea trasversale. Mi causa dolore acerbissimo e non mi lascia prendere un po' di riposo. Cosa è mai questo? Questo fenomeno nuovo incominciai ad avvertirlo dietro un'altra apparizione di quel solito misterioso personaggio del cinque e sei agosto e del 20 ottobre [sic!], di cui vi parlai, se vi rammentate, in altre mie lettere ${ }^{119}$.

Lautore della lettera, padre Pio, parla della transverberazione fisica del suo cuore, questa volta è una "lamina di ferro che dalla parte bassa del cuore si estende sino a sotto la spalla destra in linea trasversale", portando un "dolore acerbissimo" e continuo. La lettera tratta dello stesso "personaggio misterioso" e di un "fenomeno nuovo" in stretta connessione con quello dell'agosto precedente, che indica alla transverberazione "del cinque e sei agosto" e la stimmatizzazione "del 20 ottobre [sic!]"120. Il fenomeno è nuovo perché fino allora non si era mai verifi-

117 Epist. I, 1103.

118 Carmelitano, Padre Roberto Moretti, prof. dell'Istituto Pontificio di Spiritualità del Teresianum in Roma, conferma che esiste qualche testimonianza della transverberazione del cuore di padre Pio, ma questi testi, secondo lui, parlano del dolore spirituale, della ferita dell'anima, perciò confessa che personalmente non è convinto della sua realtà fisica; Cf. R. Moretti, La transverberazione di Padre Pio, [in:] Atti del $1^{\circ}$ Convegno di studi sulla spiritualità di Padre Pio (San Giovanni Rotondo, 1-6 maggio 1972), a cura di G. Di Flumeri, San Giovanni Rotondo 1973, p. 317.

119 Epist. I, 1106.

120 Evidentemente si riferisce al 20 settembre del 1918. 
cato in padre Pio e la ferita possiede la natura fisica ${ }^{121}$ non spirituale, di cui, precedentemente, parecchie volte egli aveva parlato ${ }^{122}$.

Non è facile avere descrizione dettagliate sulla configurazione fisica della transverberazione, anche se si rende visibile nella carne, data la natura intima del fenomeno. Generalmente non è oggetto d'osservazione diretta, ma si può affermare che indubbiamente il corpo partecipa al dolore della piaga sia al costato, sia al cuore. Il protagonista del mirabile fenomeno del transverberazione sente fortemente il dolore fisico causato dalle piaghe sul suo corpo. Egli usa la terminologia che non è solamente spirituale, simbolica o metaforica, ma viene dal vocabolario delle espressioni realistiche. Il dolore fisico è reale e ha un valore di conformazione al Cristo crocifisso.

\section{Le stimmate}

Dopo aver presentato la transverberazione del costato e del cuore di padre Pio, possiamo passare ad analizzare un'altra grazia speciale, la stimmatizzazione. Il fatto notevole è che scienza psichiatrica e teologica convengono sul fatto che le stimmate sono un singolare linguaggio del corpo ${ }^{123}$, che s'inserisce nei complessi rapporti tra anima e corpo. La stimmatizzazione è il fenomeno mistico che ha reso noto padre Pio e lo ha portato alla ribalta della cronaca del tempo e della popolarità, d'altronde era "il dono mistico più scomodo, alla luce dei provvedimenti ecclesiastici e delle sofferenze causategli per umiliazione e pregiudizi, manifestazioni fanatiche di vario tipo e provenienza, incomprensioni ed isolamenti" 124 , come abbiamo già scritto nel paragrafo sul Sant'Offizio. La stimmatizzazione di padre Pio ha avuto luogo in due tempi, tra gli anni 1910-1918: la prima in modo invisibile e la seconda in modo visi-

121 Padre Gerardo Di Flumeri afferma che "la ferita, che noi riteniamo fisica, dovette spaccare il cuore da parte a parte, in due, iniziando da sinistra (in basso) e andando verso destra (in alto)", G. Di Flumeri, La transverberazione di padre Pio da Pietrelcina, 53.

122 Cf. Epist. I, 300; 366; 522; 1103.

123 Cf. Introduzione, [in:] Padre Pio. Le mie stimmate. Le lettere del santo di Pietrelcina, a cura di G. Pasquale, Milano 2002, p. 17.

${ }_{124}$ Cf. L. Lavecchia, L'itinerario di fede di Padre Pio da Pietrelcina nell'Epistolario, 179. 
bile. Il religioso dà la testimonianza del primo tipo di stimmatizzazione nella lettera a padre Benedetto l'8 settembre del 1911, riportandola nei seguenti termini:

Ieri sera poi mi è successo una cosa che io non so né spiegare e né comprendere. In mezzo alla palma delle mani è apparso un po' di rosso quasi quanto la forma di un centesimo, accompagnato anche da un forte ed acuto dolore in mezzo a quel po' di rosso. Questo dolore era più sensibile in mezzo alla mano sinistra, tanto che dura ancora. Anche sotto i piedi avverto un po' di dolore. Questo fenomeno è quasi da un anno che si va ripetendo, però adesso era da un pezzo che più non si ripeteva ${ }^{125}$.

Padre Pio parla del fenomeno in modo immediato, semplice e puntuale. La descrizione mostra che le mani e i piedi sono interessati da un certo dolore, che è più acuto nelle mani, ove è visibile un po' di rossore, "quasi quanto la forma di un centesimo", non costantemente avvertito nell'arco di un anno: "era da un pezzo che più non si ripeteva".

Le stimmate invisibili diventano visibili perennemente dal 20 settembre 1918. Padre Pio nella lettera del 22 ottobre successivo, indirizzata al suo direttore spirituale, fa l'ampio resoconto di questo fenomeno:

Cosa dirvi a riguardo di ciò che mi dimandate del come sia avvenuta la mia crocifissione? [...] Era la mattina del 20 dello scorso mese in coro, dopo la celebrazione della santa messa, allorché venni sorpreso dal riposo, simile ad un dolce sonno. [...] E mentre tutto questo si andava operando, mi vidi dinanzi un misterioso personaggio, simile a quello visto la sera del 5 agosto, che differenziava in questo solamente che aveva le mani ed i piedi ed il costo che grondava sangue. [...] La vista del personaggio si ritira ed io mi avvidi che mani, piedi e costato erano traforati e grondavano sangue. Immaginate lo strazio che esperimentai allora e che vado esperimentando continuamente quasi tutti i giorni. La ferita del cuore gitta assiduamente del sangue, specie dal giovedì a sera sino al sabato. Padre mio, io muoio di dolore per lo strazio, non il dolore perché lo veggo impossibile ed io sento di volermi inebriare di dolore [...]. Il personaggio di cui intendevo parlare nell'altra mia precedente non è altro che quello stesso di cui vi parlai in un'altra mia, visto il 5 agosto. Egli segue la sua operazione senza posa, con superlativo strazio dell'anima. Io sento nell'interno un continuo rumoreggiare, simile ad una cascata, che gitta sempre sangue ${ }^{126}$.

Il religioso stigmatizzato, in modo molto suggestivo, descrive la sua mistica crocifissione, avvenuta nel coro sopraelevato alla porta d'in-

125 Epist. I, 234.

126 Epist. I, 1093-1095. 
gresso della chiesa, di venerdì, davanti al crocifisso Seicentesco, dopo la celebrazione della messa nella chiesa di Santa Maria delle Grazie del convento a San Giovani Rotondo. L'operazione è tutta divina ed è posta in continuità con quella del 5 agosto, per via della presenza dello stesso personaggio misterioso: "Egli segue la sua operazione senza posa".

Il racconto di padre Pio relativo alla stimmatizzazione del 20 settembre 1918 evidenzia i seguenti elementi: apparizione di un personaggio misterioso grondante sangue; riscontro delle mani e dei piedi perforati; dolore e timore; invocazione della scomparsa dei segni, ma non del dolore. Le ferite dolorose e grondanti sangue alle mani, ai piedi, al costato e al cuore sono la conformazione crocifissa a Cristo e l'associazione alla sua passione, per la vocazione-missione "corredentrice"127. La sofferenza interiore di padre Pio si accompagna con piaghe esterne, come le stimmate autentiche ${ }^{128}$. Il fenomeno delle stimmate chiude la nostra riflessione sul tema del dolore fisico "sopranaturale". In conclusione, nella prima parte del secondo paragrafo del presente capitolo ci siamo interessati al fenomeno del dolore fisico di padre Pio in vari settori. All'inizio abbiamo presentato i dolori fisici naturali, mettendo in rilievo la salute e le malattie del religioso; in seguito, abbiamo considerato i dolori fisici "sopranaturali", provandoli a dividere in due classi: quelli d'origine diabolica, con il dolore delle vessazioni demoniache e quelle d'origine divina, con il dolore della transverberazione del costato, del cuore e quello delle stimmate.

\subsection{La sofferenza spirituale o morale}

La sofferenza o il dolore spirituale o morale è un patire che non lascia tracce esterne, ma che disegna un cammino invisibile che possiamo chiamare "via crucis". Le dichiarazioni che ci offre padre Pio delle

${ }_{127}$ Cf. L. Lavecchia, L'itinerario di fede di Padre Pio da Pietrelcina nell'Epistolario, 182.

${ }^{128}$ In merito all'autenticità delle stimmate, disponiamo di diverse deposizioni lasciateci da testi autorevoli, che spaziano della medicina, sino a, quel gerarchico religioso ed ecclesiastico, per terminare con quello affettivo ed occasionale. I dottori interpellati nell'immediato manifestarsi delle stimmate furono: Luigi Romanelli, Amigo Bignami e Giorgio Festa; Cf. BD I, 169174; Cf. G. Cruchon, Le stimmate di Padre Pio, in Convegno I, 101-129. 
sue passioni morali non sono soltanto descrittive, ma esprimono tutto un lungo itinerario di perfezionamento interiore. Parte dalle tentazioni più sensibili, quelle dei dubbi e gli scrupoli, passando per quelle pene più amare come i timori di essere un illuso e di poter essere allontanato da Dio, finendo poi con le lotte per la difesa della sua fede e la fiducia in Dio. Tutto questo quadro oscuro del dolore e della sofferenza spirituale di padre Pio è senza dubbio illuminato attraverso le consolazioni e le grazie speciali, pur essendo preso dalla "notte oscura" che prevaleva nel mistero della sua vita. Il presente paragrafo accanto alla descrizione di sofferenza spirituale "in genere" ci fa vedere la sofferenza spirituale particolare, cioè la "notte oscura" ed alcune cause delle sofferenze spirituali come timori e tentazioni.

I fenomeni descritti i dolori fisici "sopranaturali", le vessazioni diaboliche, la transverberazione del costato e del cuore e le stimmate, causano la sofferenza morale, quella spirituale oppure entrambe insieme. Le sofferenze morali come le tentazioni diaboliche le presenteremo nel capitolo seguente, mentre delle sofferenze spirituali delle ferite d'amore abbiamo accennato qualcosa precedentemente, discutendo il dolore fisico dei fenomeni mistici. Vogliamo quindi indicare la "notte oscura" come uno dei più importanti sintomi della sofferenza spirituale. Il nostro compito non è però la disquisizione sul fenomeno della sofferenza, che consta di tre specie differenti, ma provare a descriverle e classificarle. Il presente paragrafo illustra il vocabolario inerente la sofferenza spirituale di padre Pio "in genere".

All'inizio trattiamo del religioso che soffre fisicamente e spiritualmente dolori ormai non più sopportabili, peraltro più intensi nello spirito: "Le lotte spirituali, in paragone di ciò che vado soffrendo nel corpo, sono assai superiori, sebbene anche le sofferenze corporali si vanno rendendo sempre maggiori"129. La partecipazione del corpo alle sofferenze spirituali è meglio precisata in una lettera del 24 febbraio 1911 indirizzata a padre Benedetto: "Riguardo alle afflizioni e guerre spirituali, l'assicuro che vanno di pari passo con l'afflizioni corporali. Al moltipli-

Epist. I, 212-213. 
carsi di queste succede l'aumento di quelle"130. Il testo mostra la simultanea esistenza e la reciproca infiltrazione del dolore fisico e della sofferenza spirituale.

Padre Pio quando parla di sofferenza spirituale, come il "dolore dell'anima", lo fa distinguendola da quella fisica, presente nella sua vita, e si riferisce a qualcosa di intimo, di profondo. Egli scrive a padre Benedetto: "Padre mio, aiutatemi, perché il dolore tutto spirituale, che sento è troppo intimo, è troppo sottile, è consumante"131. La parola "dolore" qui indica la sofferenza spirituale, ma di solito, il religioso spiegando lo stesso tipo di sofferenza, usa il termine "pena"132. Un altro gruppo di espressioni, utilizzate da padre Pio nel descrivere il fenomeno della sofferenza spirituale, sono gli aggettivi: tormento amaro (amarezza) ${ }^{133}$, afflizioni oltremodo ${ }^{134}$, sommamente afflitto ${ }^{135}$, afflizioni estreme ${ }^{136}$, crudele martirio ${ }^{137}$. Egli impiega metafore come nubi oscure e tenebrose ${ }^{138}$, o utilizza una terminologia bellica parlando di lotte e guerre spirituali ${ }^{139}$, spada di dolore e lagrime di dolore. Dopo aver descritto il fenomeno della sofferenza spirituale "in genere", attraverso i vocaboli utilizzati, passiamo all'analisi particolare della sofferenza spirituale e morale di padre Pio.

\section{La notte oscura ${ }^{140}$}

Il presente paragrafo ci introduce il fenomeno della "notte oscura" di padre Pio, così come traspare dal suo Epistolario I. Il primo volume dell'e-

130 Epist. I, 214.

131 Epist. I, 428.

132 Epist. I, 367; 384; 421; 469.

133 Epist. I, 427, 469.

134 Epist. I, 185; 203; 226.

135 Epist. I, 394.

136 Epist. I, 469.

137 Epist. I, 383.

138 Epist. I, 469.

139 Epist. I, 212; 214.

140 l termine "notte oscura" non è inventato da Giovanni della Croce, anche se è lui a fornirgli diffusione e fama, ma è ripreso dalla tradizione mistica, in particolare da Gregorio Nisseno, dallo Pseudo-Dionigi e da Taulero. Tuttavia fu Giovanni della Croce ad attribuirgli quel valore centrale che ne fa l'espressione sintetica dell'esperienza mistica. Su di essa ci sono vari frainten- 
pistolario abbraccia soltanto dodici anni (1910-1912), per il noto decreto del Sant'offizio, che proibiva al religioso stigmatizzato di rispondere alle lettere ricevute, perciò il raccolto delle lettere ci rivela alcune tappe della sua vita mistica. Le dure prove dello spirito che accompagnano il religioso e la purificazione passiva dell'anima nella via della perfezione portano la forte sofferenza spirituale. Si tratta di aridità, desolazioni e di dolorosissime operazioni che Dio compie nell'anima, alle quali però non manca di parteciparvi, intensamente, anche il corpo. E da questa prospettiva nelle lettere di padre Pio appare un dolore profondo che sale prima a Dio e poi al padre spirituale.

Le prime lettere contenute nell'Epistolario I non riguardano immediatamente la "notte oscura", però, con la lettera del giugno 1913, lo scenario cambia e si percepisce chiaramente l'inaugurazione di una nuova fase mistica. Dice padre Pio:

L'anima mia che sempre più va ricevendo favori da Dio non è soddisfatta, che anzi geme sempre e sospira perché sempre le si raddoppia il dolore. Di giorno in giorno a lei si scoprono sempre più le grandezze del suo Dio ed a tale luce, che sempre più viva in lei si va facendo, arde dal desiderio di a lui unirsi con vincoli indissolubili. Vede in questa luce quanto questo adorabile Signore merita d'essere amato e si sente sempre più infiammata per lui. Ma, oh Dio!, questo stesso desiderio di unirsi a lui, di amarlo quanto ne può una creatura, le producono una gran pena vedendo sempre più quanto è lei separata e lontana dal possederlo senza timore di perderlo ${ }^{141}$.

Il testo evidenza un contrasto tra i favori ricevuti da Dio, la scoperta delle sue grandezze e il dolore determinatosi per il desiderio dell'anima di unirsi a lui "con vincoli indissolubili", che rimane deluso a causa della separazione e dell'incapacità di "possederlo senza timore di perderlo". Questa dinamica, ove da "una parte vi è la comunicazione di Dio

dimenti, il più frequente dei quali è quello di identificare notte oscura con sofferenza e nient'altro, allorché l'anima si unisce a Dio "trasformata dall'amore". Inoltre questa notte ha due modalità, che sono l'attiva e la passiva, la prima fatta di opportuni sforzi da parte dell'interessato, la seconda data per grazia. Si tratta dunque di una progressiva trasformazione, che è una purificazione del soggetto, il quale perde uno dopo l'altro i suoi attaccamenti ai sensi e alle facoltà psichiche (intelletto, immaginazione e desiderio). Cf. San Giovanni della Croce, Salita del Monte Carmelo, [in:] Opere, $6^{\text {a }}$ ed., Roma 1991, pp. 410-411; Cf. H. U. von Balthasar, Gloria. Una estetica teologica. Stili laicali (Volume terzo), Milano 1985, pp. 99-107.

141 Epist. I, 366. 
e dall'altra la lontananza da Dio, conduce gradualmente nella sua evoluzione alla notte dello spirito" ${ }^{142}$. Vedere l'applicazione di tutto ciò in padre Pio significherebbe analizzare molte lettere, da parte nostra opereremo una selezione, privilegiando quelle che ci sono sembrate più paradigmatiche, in cui appare la sofferenza spirituale e che sono indirizzate a padre Benedetto.

Con chiarezza di concetti il religioso spiega a padre Benedetto il fenomeno che ormai si andava sviluppando nella sua anima. Nella lettera del 4 maggio del 1914, scrive:

E' l'altra notte per l'anima. L'anima viene posta in afflizioni estreme e pene interiori di morte. La divina bontà viene rappresentata all'anima in tal modo, da non poter la poverina godere di tanta bontà con possedimento di amore, ma glielo lascia sospirare solo da lunghi un tal possesso con desideri estremamente penosi; l'anima posta in questo stato anela a Dio, fonte di ogni bene, e solo con un profluvio di lacrime palesa la pena delle sue brame. [...] l'anima stessa sembra che si sia votata a congiurare contro se stessa servendosi a tal fine della fantasia e della immaginazione. I belli giorni passati col dolcissimo Gesù spariscono del tutto dalla mente. Non può l'anima, posta in tal stato, fare a meno di esclamare: "Per me tutto è perduto!". Lo strazio che prova la poverina è tale che non saprei differenziarlo dalle pene atrocissime che soffrono i dannati nell'inferno ${ }^{143}$.

Padre Pio fa capire che si trova nell'alta "notte oscura" per l'anima e ne spiega le manifestazioni: estrema sofferenza spirituale, vivo e doloroso desiderio di Dio determinato dalla rappresentazione della sua bontà nell'anima, incapacità ad avere un contatto, fantasie e immaginazioni disturbanti, sensazioni di disperazione uguali alle "pene che soffrono i dannati nell'inferno"144.

142 Cf. L. Lavecchia, L'itinerario di fede di Padre Pio da Pietrelcina nell'Epistolario, 30.

143 Epist. I, 469.

144 Padre pio fa il richiamo alle pene infernali come termine di paragone delle sofferenze che sta soffrendo. L'insistenza di questa componente è spiegata da san Giovanni della Croce, quando dice: "La maggiore sofferenza provata dall'anima in questo stato è dovuta al fatto che ella è convinta che Dio l'abbia riprovata e, aborrendola, l'abbia gettata nelle tenebre; per lei infatti è grave e dolorosa pena il pensiero di essere abbandonata dal signore [...] sente molto vivamente [...] i dolori dell'inferno, che consistono nel sentirsi senza Dio [...] Tutto questo l'anima sente in tale stato, tanto più gravemente, poiché le pare che ciò debba durare per sempre". Cf. San Giovanni della Croce, Salita del Monte Carmelo, [in:] Opere, 6a ed., Roma, 1991, p. 410-411. 
Le pagine dell'Epistolario I, che vanno dal 1916 al 1922 sono dense di testimonianze della terribile prova che si intensifica e di descrizioni delle sofferenze spirituali e morali che continuano ad essere le "coordinate" della nuova tappa. La lettera successiva, indirizzata a padre Benedetto, che andiamo ad analizzare porta la data del 6 settembre 1916. Ne presentiamo un corto brano:

Sono messo alla prova di tutto. Vivo in una perpetua notte e questa notte non accenna affatto di ritirare le sue folte tenebre per dar luogo alla bella aurora. Iddio lo sento nel centro dell'anima mia, ma non saprei dirvi il come lo sento. Questa di lui presenza, lunghi dal consolarmi, accresce all'infinito il mio martirio, che in ogni istante mi fa cadere in un deliquio estremamente penoso. [...] l'oggetto da contemplare si va sempre più ingrandendo, tenendosi sempre più nascosto ${ }^{145}$.

Questa descrizione dello stato spirituale dell'anima di padre Pio indica che la notte diventa "perpetua", però, il religioso sente Iddio "nel centro dell'anima", ma la percezione è molto confusa, al punto che si fa apportatrice di martirio e causa di "deliquio estremamente penoso". La grazia che riceve e la sofferenza che sperimenta mettono in rilievo quello che dice alla fine: "l'oggetto da contemplare si va sempre più ingrandendo, tenendosi sempre più nascosto". Dio vuole realizzare in padre Pio questo progresso spirituale, attraverso la sofferenza che produce il suo nascondersi.

Tale stato di fatto si ripete ed è più sottolineato nella lettera datata 4 dicembre 1916. In essa padre Pio scrive così:

Sento tutto il peso della solitudine, vorrei trovare una persona capace di ricevere tutte le ambasce, che si vanno aggirando nel mio animo. Ma, padre mio, dove trovarla? Io mi sento solo a combattere, di giorno e di notte, e non mi accorgo di altro se non che della mano di Dio, che si va sempre più aggravando sopra di me. A me sembra che Iddio si sia ritirato completamente e non mi fa esperimentare altro, se no la mia estrema miseria. Il nemico mi tenta su tutto e vuol darmi a credere che tutto sia finalmente finito per $\mathrm{me}^{146}$.

L'autore della lettera si lamenta, perché sente la forte solitudine e non può trovare neppure una persona su cui riversare le sue ambasce. Egli,

145 Epist. I, 817-818.

146 Epist. I, 849; Cf. Epist. I, 858; 873; 882; 904; 947; 978; 992-993. 
allo stesso tempo, sente la crudele tentazione del demonio. L'esperienza dell'abbandono, l'intervento diabolico e la già descritta sofferenza delle pene che "soffrono i dannati nell'inferno" hanno il senso purificatore. Proseguendo cronologicamente nell'indagine sull'Epistolario I del nostro autore giungiamo alla lettera del 4 giugno del 1918, scritta a padre Benedetto. In essa leggiamo:

Da giovedì al giorno mi sento più che mai riempito l'anima di un'estrema turbazione. Sento che la mano del Signore si è aggravata su di me, sento che il Signore va addimostrando tutta la sua potenza nel punirmi, e come foglia rapita dal vento, egli mi rigetta e mi perseguita. [...] Mio Dio, sono smarrito e ti ho perduto, ma ti ritroverò? Oppure ti avrò smarrito per sempre? Mi hai condannato a vivere eternamente lontano dalla tua faccia?... Padre mio, mi vado addentrando come posso in questa oscura prigione, ma è arduo l'avanzare tra il fuoco buio di queste fitte tenebre, tra l'uragano ed il rimestar di vessazione nemica, che approfitta della tempesta per farmi prevaricare e vincermi. [...] La mia preghiera poi, padre mio, è l'aculeo di dolori e di pene mortali, orribile a ripensarsi. Io non raccapezzo più nulla, non so se le mie preghiere siano tali, oppure forti risentimenti che il cuore rivolge al suo Dio nella piena del suo dolore. Sento un abbandono di vuoto in me, orribile a ripensarsi quando si è in esso. Nulla, il perfetto nulla, fuori dei lampi rarissimi guizzanti di luce incerta, tra le forte tenebre in cui si è immerso, che dicono allo spirito: Dio è nel bene. Ma Dio è sempre occulto all'attento spirito, che si consuma vegliando in affannose, ma sempre necessarie ricerche: ed il povero spirito si va consumando in tanti timori di offenderlo, dato che è solo in solitudine desolatissima ${ }^{147}$.

Il tenore della lettera, sottolineando la drammaticità del momento, ci dà la descrizione di uno scenario più calcato nelle tinte oscure e più arricchito degli elementi di sofferenza. Lo stato di desolazione, il senso di abbandono e l'idea chiara del rifiuto di Dio sono più accentuati. "Padre Pio vive la caligine del nascondimento di Dio e del suo smarrimento, a cui fa eco una sorta di elegia per l'amore perduto"148. La "notte oscura" nel cammino di perfezione viene vissuta da padre Pio come un sentirsi avvolti da dense e tormentose caligini, che impediscono la visione e la comprensione e determinano smarrimento, timore, ansia, pianto e soprattutto sofferenze spirituali. Questi elementi creano una notte dentro e attorno al dolente religioso, che con la fiducia e la "nuda" fede proce-

147 Epist. I, 1027-129; Cf. Epist. I, 1033-141; 1049; 1050-1053; 1062-1065.

148 L. Lavecchia, L'itinerario di fede di Padre Pio da Pietrelcina nell'Epistolario, 37. 
de in questa oscurità profonda senza interrompere il suo cammino spirituale. Le cause che provocavano le sofferenze spirituali di padre Pio erano dappertutto i timori e le tentazioni. Certi timori in sé stessi vengono considerati anche tentazioni, nella misura in cui si percepisce che sono il frutto delle suggestioni diaboliche.

\section{I timori}

La prima causa della sofferenza spirituale di padre Pio che ci appare è il timore. L'Epistolario I ci rivela i motivi dei suoi timori, che noi raggruppiamo in due tipi: timori verso le sue confessioni e suoi peccati; timore di essere ingannato oppure di ingannare. Nel gruppo delle lettere, dal 20 giugno del 1910 al 4 maggio del 1914, i timori che soffre padre Pio hanno le proprie caratteristica dell'anima, che progredisce decisamente sul cammino della perfezion $\mathrm{e}^{149}$. All'inizio, mettiamo in luce il dolore che sente Padre Pio per aver detto una bugia. Il timore tocca il peccato, in cui crede di esser caduto. Egli scrive nel settembre del 1911 al suo direttore spirituale:

Mio caro padre, col sangue agli occhi scrivo la presente. Sappia, padre mio, che giorni or sono ho avuto il coraggio di nuovamente offendere Gesù con una bugia. Mio Dio che vergogna ${ }^{150}$.

In questo stadio purgativo incontriamo i timori o gli scrupoli sulla validità delle confessioni, dei rimorsi per la sua vita passata e il timore che quello che gli succede non sia di natura sopranaturale, ma pura illusione.

Padre Pio sente i timori più forti verso le sue confessioni e i suoi peccati quando si converte al Signore. Egli ne dà la testimonianza, quando scrive a padre Benedetto:

Ascolti, padre mio. Da vari giorni mi sento un continuo turbamento di coscienza per la mia vita passata, spesa così malamente. Ma ciò che in particolare più mi martirizza il cuore e mi affligge oltremodo si è il pensiero di non essere certo di aver confessati tutti i peccati della mia vita passata e quello ancora di averli o no confessati bene ${ }^{151}$.

149 Cf. A. Queralt, El «dolor»: su valor cristologico. Estudio teologico-spiritual del autotestimonio del P. Pio de Pietrelcina, O.F.M.Cap., in QS, 153.

150 Epist. I, 235.

151 Epist. I, 185. 
Egli si sente martirizzato e afflitto "oltremodo" a causa dei rimorsi per non "aver confessati tutti i peccati della vita passata" e non sa se "averli o no confessati bene". Il tema della confessione si ripete: padre Pio chiede al suo direttore spirituale il permesso di una "bella confessione generale"152. La risposta, che scrive padre Benedetto è negativa ${ }^{153}$, e nella seguente lettera, del 7 maggio del 1911, lo afferma:

\begin{abstract}
Mio carissimo Pio, tu non pecchi, hai capito? E perciò non sei tenuto a confessarti necessariamente delle tentazioni. Solo per spirito di umiltà le puoi rivelare e non ti devi confondere a sapere e conoscere se hai acconsentito o no. Ripeto dunque che non vi è peccato alcuno in queste battaglie, ma solo palme e corone $[. . .]^{154}$.
\end{abstract}

Però, la categorica risposta del direttore spirituale con la sua garanzia che il suo discepolo non ha peccato, non toglie il timore. Padre Pio scrive di nuovo. In una lettera datata il 2 settembre 1911 egli confessa:

Alle volte mi viene una disperazione grande perché mi sembra quasi impossibile che Gesù debba perdonarmi tanti peccati; il più delle volte sembrami impossibile che Gesù voglia perdonarmi ${ }^{155}$.

Il timore, nato dal dubbio che i peccati siano stati perdonati da Gesù, paralizza il religioso. Egli si batte tra il timore e la speranza di essere perdonato, oppure quello di rimanere nella riprovazione del Signore. La successiva risposta categorica di padre Benedetto, cui afferma che egli non pecca, non gli dà pace interiore. Il direttore scrive: "Caro Pio, non è vero che pecchi; non è vero che dai disgusto al Signore e perciò non è vero che il Signore non ti perdonerà. La grazia divina è con te e molto tu sei caro al Signore"156.

Oltre tutto, nel 1912 appare ancora la stessa domanda: egli chiede se deve confessare tutti i "peccati dubbi"157. In questo quesito si percepisce l'angoscia propria di padre Pio che è sottomesso agli scrupoli. Altro tipo di timore del religioso da Pietrelcina è quello di essere ingannato

\footnotetext{
152 Epist. I, 219.

153 Epist. I, 221.

154 Epist. I, 222.

155 Epist. I, 231.

156 Epist. I, 232.

157 Epist. I, 278.
} 
dal Signore e vivere una pura illusione; e non soltanto di essere ingannato, ma anche di ingannare i suoi direttori spirituali. Nella lettera, del 1 novembre del 1913, a padre Benedetto confessa:

Mi assale una profonda tristezza ed un pensiero atroce mi attraversa per la mente, quello cioè di poter essere un illuso senza conoscerlo. Dio solo sa che tormento è questo per me! Forse che il Signore, mi penso, in pena delle mie infedeltà non potrà permettere che io, senza saperlo, ingannassi me stesso ed i direttori del mio spirito ${ }^{158}$.

L'autore della lettera tristemente scrive del suo tormento che sente a causa della possibilità di essere illuso. Intuisce il possibile fondamento di questa situazione permessa dal Signore, come una forma per punire i suoi peccati e come castigo degli stessi. A questi timori aggiunge anche quello di essere ingannato dal demonio. Padre Pio comunica al suo direttore spirituale: "Temo fortemente l'inganno del nemico e chi sa, me ne vado pensando, che non sia io veramente nella rete del nemico"159. Con questo riferimento all'intervento demoniaco, che padre Pio collega al suo timore di essere illuso, passiamo alla causa seguente della sofferenza spirituale: le tentazioni.

\section{Le tentazioni}

Non abbiamo le definizioni precise delle tentazioni, ma un vocabolario generico che esprime queste situazioni, che possiamo ritrovare nell'Epistolario I. Padre Pio spiega che le tentazioni "sono più che mai accanite contro di me" ${ }^{160}$, che il nemico lo angoscia con "fantasmi e spauracchi" e mette veleno nella sua vita con "infernali insidie" ${ }^{161}$. Il religioso da Pietrelcina si trova sottomesso a tensioni interiori forti, soprattutto quelle causate dalle tentazioni paradossalmente dovute alle grazie speciali ricevute, sebbene sia consapevole delle limitazioni delle sue forze per resistere o perseverare. Questa sua debolezza è fisica e morale. Fisica come conseguenza della sofferenza provocata dalle tentazioni.

\footnotetext{
158 Epist. I, 424.

159 Epist. I, 590.

160 Epist. I, 203.

161 Epist. I, 314.
} 
Nella lettera del 9 aprile del 1911 indirizzata a padre Benedetto, il religioso descrive la sofferenza delle tentazioni:

Quindi sono solo a combattere e mi trovo col cuore ripieno di spavento. Che mi accadrà non lo so. Mi sento debole assai di anima e di corpo, padre mio, ma mi abbandono in Dio. Se ciò è volere di Dio voglio patire e sempre più per dargli gusto ${ }^{162}$.

Lo "spavento" del quale si sente pieno poiché ignora "che accadrà", lo indebolisce nel corpo e nell'anima. Primo scopo del demonio, in questa lotta spietata, è quello di generare nell'anima uno stato di diffidenza e di sfiducia nella misericordia divina. Nella lettera del 17 agosto del 1910 padre Pio scrive così: "È anche vero però che il demonio non può darsi requie per farmi perdere la pace dell'anima e scemare in me quella tanta fiducia, che ho nella divina misericordia"163. In questo periodo soffre "per bruttezza e continua ostilità" delle tentazioni, che mostrano meglio il ruolo di Satana nel rapporto di comunione instauratosi tra Dio e lui.

Padre Pio non indietreggia né si dà per vinto. La sofferenza più atroce è causata dalla incertezza di rigettare veramente le lusinghe diaboliche. Nella lettera datata il 6 luglio 1910 confessa: "Dietro le innumerevoli tentazioni, alle quali vado soggetto di giorno in giorno, un dubbio da sconvolgermi anche la mente mi rimane: se veramente le ho discacciate"164. Il religioso sperimenta il dubbio e lo scrupolo: questi sono i pungoli diabolici nel suo processo della purificazione. In questa fase il nemico cerca di far perdere la speranza a padre Pio, e vuole "strapparlo" dalle braccia di Dio, ma il religioso, invece, fa forte resistenza alle sue tentazioni. Possiamo dire che questa disperazione viene causata dal ricordo della sua vita passata.

La lettera che segue spiega il modo con cui il demonio mette in crisi il cuore del cappuccino. Era il 19 marzo 1911.

Le tentazioni che riguardano la mia vita secolare son esse che più mi toccano il cuore, mi offuscano la mente, mi fanno sudar freddo e sarei per dire mi fanno tremare da capo a piedi. In tali momenti gli occhi solo mi rimangono per piangere; e mi

\footnotetext{
162 Epist. I, 219.

163 Epist. I, 196.

164 Epist. I, 187.
} 


\section{Błażej Strzechmiński OFMCap}

vado confrontando ed incoraggiando solo pensando a ciò che lei mi va suggerendo nelle sue lettere ${ }^{165}$.

Le tentazioni che fanno più presa nel cuore del religioso sono quelle riguardanti la vita secolare che, accanto alla sofferenza morale, mostrano gli effetti della sofferenza fisica: "fanno sudar freddo" e "fanno tremare da capo a piedi”. Un'altra lettera indirizzata a padre Benedetto, del 9 aprile dello stesso anno, ci informa che:

[...] il nemico anche in questi giorni santi fa tutti i suoi sforzi per indurmi ad acconsentire ai suoi empi disegni; ed in ispecial modo questo spirito maligno cerca con ogni sorta di fantasmi d'introdurmi nel cuore pensieri d'immondezza e di disperazione ${ }^{166}$.

Padre Pio più precisamente parla di introduzione di "pensieri d'immondezza e di disperazione”. E' provato dal nemico dell'anima nei sensi e nello spirito. Attorno all'anno 1915 le tentazioni si rafforzano, aumentando anche il suo timore di essere vinto. Nello stesso anno lui sottolinea il dolore del quale è vittima e che lo fa arrivare ai suoi limiti, un dolore fortissimo, quello che gli fa desiderare la morte come sollie$\mathrm{vo}^{167}$. Vedeva cosi la morte come rimedio alla sua sofferenza, come una liberazione, e chiede che il Signore disponga della sua vita ${ }^{168}$. Il religioso nello stesso tempo soffre tentazioni contro la fede e pensieri di bestemmia. Scrive al suo direttore spirituale: "Ci sono poi certi momenti che vengo assalito da violente tentazioni contro la fede" 169 , e in un'altra lettera: "Pensieri di bestemmie mi attraversarono di continuo la mia mente" 170 . Attraverso il primo tipo di tentazione, il demonio vuole distruggere la fiducia in Dio e fa considerare il peccato come un fatto dilettevole; ed attraverso le tentazioni di bestemmie cerca d'inserirsi nel rapporto d'amore, di profonda lode ed adorazione esistente tra padre Pio ed il suo Signore.

165 Epist. I, 215-216.

166 Epist. I, 219; Cf. Epist. I, 224; 226.

167 Cf. Epist. I, 521-522.

168 Cf. A. Queralt, El «dolor»: su valor cristologico. Estudio teologico-spiritual del autotestimonio del P. Pio de Pietrelcina, O.F.M.Cap., [in:] QS, 161.

169 Epist. I, 910.

170 Epist. I, 838. 
Nell'Epistolario I è viva la voce silenziosa del rapporto vita e morale. La totalità che riempie l'anima è anche una forza morale, per la quale l'anima percepisce nella pienezza la propria miseria e scopre la divina misericordia. Alle sofferenze fisiche di padre Pio a causa degli assalti del demonio, che abbiamo già presentato sopra, si aggiungono le tentazioni, come la dura prova e come la sofferenza spirituale nel processo di purificazione dei sensi e dello spirito. Il demonio attraverso le tentazioni avrebbe voluto portare il cappuccino alla distruzione della pace, della fiducia e della comunione con Dio. Nel confronto col diavolo si evidenzia da parte del religioso la virtù della fede che ha sempre funto da sua propria opzione fondamentale a fronte di ogni proposta diabolica. Con questa paragone finisce la nostra riflessione sul tema della sofferenza spirituale e morale di padre Pio. Concludendo la seconda parte del paragrafo sul fenomeno della sofferenza, possiamo dire che questa realtà, cioè la sofferenza spirituale e morale, di cui abbiamo trattato è molto ricca e insieme più difficile da presentare. Nel nostro studio siamo riusciti a mostrare tre tipologie di sofferenza: il primo, la sofferenza spirituale "in genere"; il secondo, la "notte oscura" e il terzo, alcune cause della sofferenza spirituale.

Possiamo ora formulare alcune nostre conclusioni cercando di riassumere il quadro della sofferenza di padre Pio, come ci mostra l'Epistolario I. Corrispondenza con i direttori spirituali (1910-1922). In primo luogo l'Epistolario I è un volume chiave per la conoscenza di padre Pio, perciò, è la buona testimonianza della sua propria e distintiva personalità, le sue caratteristiche psico-fisiologiche, che ci mostrano la sua ricchezza affettiva, ci presentano il suo sistema nervoso molto sensibile e notevolmente delicato, e soprattutto le sue sofferenze, così spesso presenti nelle sue lettere. L'epistolario evidenzia la notevole capacità di scrittore di padre Pio, egli possiede "lo spirito del letterato, l'animo dell'artista, il cuore del poeta" e ci mostra la ricchezza dei suoi pensieri e la profondità delle esperienze mistiche.

La corrispondenza epistolare tra il cappuccino e il destinatario delle sue lettere, padre Benedetto da San Marco in Lamis, rivela il ruolo delica- 
to e molto importante, a volte quasi determinante, del direttore spirituale di padre Pio. L'epistolario contiene anche informazioni sul modo, sui motivi delle lettere stesse e la frequenza dei rapporti intercorsi tra di loro. L'Epistolario I, come abbiamo già detto, è una letteratura sulla sofferenza che offre un itinerario spirituale di padre Pio. La sofferenza dal punto di vista degli effetti che produce si manifesta nelle lettere del cappuccino come dolore fisico e dolore spirituale. Il dolore fisico ha due cause: una naturale e una "sopranaturale". La prima riguarda l'infermità o la disfunzione di qualche organo, producendo le diverse sofferenze. Nella seconda i dolori sono attribuiti a un'ingerenza diabolica o a un particolare intervento di Dio per mettere alla prova la sua fede. Molte sono le lettere nelle quali il malato religioso informa il suo direttore spirituale, padre Benedetto, delle infermità che non gli daranno tregua per tutta la vita. Padre Pio, con tutti i limiti derivanti dalle malattie e dal dolore che esse gli causano, nel senso letterale è un "corpo patologico". Come concetti conclusivi possiamo dire che quelle malattie furono reali, le loro cause furono quelle comuni della patologia umana: i fattori fisici e ambientali (freddo, caldo, umidità, ecc.), come nelle bronchiti e nei reumatismi; cariche batteriche di varie entità, come degli occhi; i sintomi clinici, quindi, non furono diversi, sul piano pratico, dalle manifestazioni e dai segni oggettivi riscontrabili, per gli stessi mali, in ogni altra persona, ma si può discutere sulla durata e sull'intensità di ciascuno di quei sintomi.

Nella scala dei dolori, proposta dal Mantegazza, possiamo affermare che padre Pio, negli ottantuno anni della sua vita, ne ha saliti praticamente tutti i gradini. Solamente negli anni 1910-1922 aveva: le algie "fredde" dell'artrosi generalizzata, i dolori bruschi e "pungenti" della polmonite, le manifestazioni "corrodenti" dell'ulcera gastrica ed i dolori "tensivi" delle emicranie, ma in tutto l'Epistolario I possiamo ancora trovare: il dolore "bruciante" della cistite emorragica, il dolore "conquassante" delle coliche renali, il dolore "contusivo" delle caviglie e dei polsi, il dolore "corrosivo" dell'epitelioma auricolare, le fitte "laceranti" dell'ernia irriducibile, il dolore "lancinanti" delle emorroidi trombizzate, il dolore "gravativo" della sinusite frontale, il dolore "terebranti" della pleurite essudativa, il dolore "pruriginoso" della pediculosi ed anche i dolori "pulsanti" degli ascessi passeggeri. Il dolore era, come s’è visto, la componente più 
illuminante della sua vita: esso non lo condizionava, né gli faceva paura. L'Epistolario I mostra gli interventi diabolici che producono i dolori fisici, come il combattimento e che portano la sofferenza morale, come le tentazioni e altre varie forme di suggerimenti che allontanano dalla volontà di Dio. Il demonio ha avuto un ruolo importante nella vita di padre Pio, si è dato molto da fare per fronteggiare la volontà del religioso, votata al Cristo e disposta al martirio, pur di conservare integra la fedeltà e confermare ed accrescere la comunione d'amore.

La descrizione dei dolori fisici "sopranaturali", come la transverberazione del costato e del cuore o le stimmate, è anche presente nelle lettere di padre Pio indirizzate al suo direttore spirituale. Il protagonista dei mirabili fenomeni sente fortemente il dolore fisico delle piaghe nel suo corpo. Il dolore fisico è reale e ha un valore di conformazione al Cristo crocifisso. La transverberazione di padre Pio è duplice: transverberazione dell'anima con effetto fisico al costato e transverberazione dell'anima con effetto fisico al cuore. La transverberazione del costato del 5-7 agosto serve a "preparare il corpo di padre Pio alla seguente stimmatizzazione", mentre la transverberazione del cuore del mese di dicembre è un "suggello d'amore" per "fare irruzione piena e definitiva dell'Amore nel suo cuore". La sofferenza o il dolore spirituale o morale è una sofferenza che non lascia tracce esterne però che disegna un cammino invisibile di perfezionamento interiore di padre Pio. Parte dalle tentazioni più sensibili, quelle dei dubbi e degli scrupoli; passa per quelle pene più amare come il timore di essere un illuso e di poter essere allontanato da Dio; continua con le lotte per la difesa della sua fede e la fiducia in Dio, soffrendo la "notte oscura" che prevale nel mistero della sua vita. Nella vita dei santi appare con tutta evidenza che Dio si serve della sofferenza, fisica e spirituale, per operare in loro il distacco dai beni terreni, lo spogliamento della volontà, la purificazione dell'anima e per preparare in loro il processo della cristificazione o affidare a loro la missione di corredenzione con Cristo. La vita del santo padre Pio da Pietrelcina ha questo carattere. 


\section{Fenomen cierpienia Ojca Pio z Pietrelciny opisany w listach do jego kierownika duchowego ojca Benedykta.}

Autor artykułu próbuje przedstawić zjawisko cierpienia, opisane przez o. Pio w jego korespondencji skierowanej do o. Benedetto z San Marco in Lamis. Analizuje je z dwóch perspektyw: jako ból fizyczny i jako cierpienie duchowe lub moralne. Wychodząc od nakreślenia bazy literackiej Epistolario I, przechodzi do opisu bólu fizycznego, klasyfikując go ze względu na przyczynę jego powstania, albo jako ból naturalny (choroby), albo ból „nadprzyrodzony”, czyli taki, którego pochodzenie jest bądź diaboliczne (dręczenie demoniczne), bądź Boże (transwerberacja boku lub serca czy stygmaty). Na koniec opisuje cierpienie duchowego i moralne, które występują podczas tzw. „nocy ciemnej” i wskazuje na niektóre z przyczyn jego powstawania (bojaźń, pokusy). Artykuł nie daje filozoficznych czy teologicznych odpowiedzi dotyczących natury cierpienia, ale analizuje fenomen cierpienia przez pryzmat efektów, jakie ono wywołuje. Jest próbą nakreślenia swoistego itinerarium duchowego o. Pio, które kształtowało się w szkole niezwykle bolesnego cierpienia, którym posłużył się Bóg dla oczyszczenia, upodobnienia i zjednoczenia go z Jego własnym Synem, Jezusem Chrystusem.

Słowa kluczowe: cierpienie, choroba, kierownik duchowy, korespondencja, noc ciemna, pokusa, stygmaty, transwerberacja, wizje diaboliczne, zdrowie fizyczne

\section{The Fenomenon of Padre Pio's suffering in the correspondence with his own spiritual director.}

Padre Pio's letters to his spiritual adviser Padre Benedetto from San Marco in Lamis provide a unique window into the spiritual life and struggle of the Saint. Into the spiritual life and struggle. Padre Pio suffered both body and spirit. In the Epistolario I he described his physical pains of different kind. He felt the natural pains caused by illness or supernatural i.e. demonic torments and of the Divine origin (transverbation, stigmata). Describing his sufferings during the "Dark Night of the Soul" he pointed out some reasons of his anxieties and temptations. In this paper, I didn't give any theological or philosophical explanation of the nature of sufferings. I d'like only to sketch a specific itinerary of Father Pio`s spiritual life. All those sufferings allowed him to purify his soul and to reach God.

Keywords: suffering, illness, spiritual director, correspondence, the dark night, temptation, stigmas, transverberation, diabolic visions, health 